\title{
Compartmental Distribution of Ventral Striatal Neurons Projecting to the Mesencephalon in the Rat
}

\author{
Henk W. Berendse, ${ }^{a}$ Henk J. Groenewegen, and Anthony H. M. Lohman \\ Department of Anatomy and Embryology, Vrije Universiteit, 1081 BT Amsterdam, The Netherlands
}

The ventral striatum is characterized by an intricate neurochemical compartmentation that is reflected in the distribution of most of its afferent fiber systems. In the present study, the compartmental relationships of ventral striatal neurons projecting to the mesencephalon were studied by combining tract tracing with the immunohistochemical localization of leu-enkephalin. Injections of the retrograde tracer cholera toxin subunit B were placed at various sites in the ventral mesencephalon. The anterograde tracer Phaseolus vulgaris leucoagglutinin was injected in single compartments in the rostrolateral part of the nucleus accumbens. The projections from the ventral striatum to the dopaminergic cell groups in the ventral mesencephalon and those to the substantia nigra pars reticulata originate from distinct subpopulations of ventral striatal neurons that respect neurochemically defined compartmental boundaries. In the "shell" of the nucleus accumbens, neurons that project to the dopaminergic cell groups are located outside areas of high cell density and weak enkephalin immunoreactivity (ENK-IR). Rostrolaterally in the "core" of the nucleus accumbens, neurons inside large areas of strong ENK-IR surrounding the anterior commissure project to the dorsomedial part of the substantia nigra pars reticulata, whereas neurons outside these areas innervate the ventral tegmental area and/or the medial part of the substantia nigra pars compacta. By contrast, more caudally in the dorsal part of the nucleus accumbens and in the ventral part of the caudate-putamen, the relationships are reversed: neurons in- or outside small patches of strong ENK-IR project respectively to the pars compacta or the pars reticulata of the substantia nigra. Since the thalamic and cortical afferents of the ventral striatum are compartmentally ordered as well, the present results imply that through the ventral striatal compartments information from disparate combinations of cortical and thalamic sources may be conveyed to distinct mesencephalic targets. The component of the ventral striatomesencephalic system reaching the dopaminergic cell groups A10, A9, and A8 may modulate the dopaminergic input to virtually the entire stria-

\footnotetext{
Received June 7, 1991; revised Dec. 30, 1991; accepted Jan. 6, 1992.

We thank Dr. Pieter Voorn for helpful discussions, Mrs. Yvonne Galis-de Graaf for technical assistance, Mr. Dirk de Jong for his photographical skills, and Ms. Loes van Zanten for secretarial support. This work was supported by NWO Program Grant 900-550-093.

Correspondence should be addressed to Henk J. Groenewegen, M.D., Ph.D. Department of Anatomy and Embryology, Vrije Universiteit, Van der Boechorststraat 7, 1081 BT Amsterdam, The Netherlands.

a Present address: Neurology Unit, University of Rochester, Monroe Community Hospital, 435 East Henrietta Road, Rochester, NY 14620.

Copyright (C) 1992 Society for Neuroscience $0270-6474 / 92 / 122079-25 \$ 05.00 / 0$
}

tum. The other component can, by way of the pars reticulata of the substantia nigra, participate in nigrothalamic and nigrotectal output pathways of the basal ganglia.

In spite of their long-standing exclusive association with motor functions, the basal ganglia are now considered as a group of interconnected nuclei that are involved in the processing of information related to many aspects of behavior, ranging from motor acts to complex emotional and motivational influences. The underlying anatomical substrate has only recently been elucidated by demonstrating a parallel organizational principle within basal ganglia connections (Heimer and Wilson, 1975; Alexander et al., 1986, 1990; Nauta, 1986). Information from functionally diverse parts of the cerebral cortex is channeled through different parts of the basal ganglia and thalamus back to the cortex, thus forming a number of parallel cortico-striatopallido-thalamo-cortical circuits that may each be involved in different aspects of behavior. The importance of this organizational principle is underscored by the parallel arrangement of the projections from the midline and intralaminar thalamic nuclei and the amygdala to both the striatum and the prefrontal cortex (Berendse and Groenewegen, 1990, 1991; Groenewegen et al., 1990b).

An important issue in basal ganglia research is whether and where integration takes place in this system of segregated circuits in order to produce coherent behavior. Local interactions may occur at the level of the striatum through overlapping inputs. Widespread integration may take place through the projections from the ventral striatum to the dopaminergic cell groups in the mesencephalon (Nauta et al., 1978; Somogyi et al., 1981; Nauta and Domesick, 1984), which seem to defy the parallel arrangement of basal ganglia connections. By way of projections to the substantia nigra pars compacta, the ventral tegmental area, and the retrorubral field, containing the A9, A10, and A8 dopaminergic cell groups, respectively (Dahlström and Fuxe, 1964; Björklund and Lindvall, 1984), the ventral striatum appears to be in a position to influence the dopaminergic input to virtually the entire striatum (Fallon and Moore, 1978; Björklund and Lindvall, 1984; Gerfen et al., 1987) and, consequently, the information processing in each of the parallel cortico-striato-pallidofugal pathways. Whether the ventral striatal projections to the dorsomedial part of the substantia nigra pars reticulata (Swanson and Cowan, 1975; Conrad and Pfaff, 1976; Nauta et al., 1978; Troiano and Siegel, 1978) originate from the same neurons remains to be established.

Superimposed upon the topographical organization of its connections, the striatum is characterized by a heterogeneous distribution of many neurotransmitters, neuromodulators, and their 
receptors. In the dorsal striatum, two neurochemically distinct compartments, the patch or striosomal compartment and the matrix compartment, have been identified (Graybiel and Ragsdale, 1978; Graybiel et al., 1981; Gerfen, 1984; Gerfen et al., 1985; for reviews, see Graybiel, 1984, 1990). Virtually all striatal afferent and efferent systems observe the boundaries of these neurochemical compartments (e.g., Herkenham and Pert, 1981; Ragsdalc and Graybiel, 1981, 1990; Gerfen, 1984, 1985, 1989; Donoghue and Herkenham, 1986; Jiménez-Castellanos and Graybiel, 1987, 1989; Giménez-Amaya and Graybiel, 1990; for reviews, see Graybiel, 1984, 1990). The differential connections of the patch/striosome and matrix compartments with the substantia nigra and associated dopaminergic cell groups constitute the most striking example of the compartmental ordering. The efferents of the striatal patches or striosomes preferentially reach the pars compacta of the substantia nigra, whereas the matrix projects mainly to the pars reticulata (Gerfen, 1984, 1985; Gerfen et al., 1985; Jiménez-Castellanos and Graybiel, 1989). The projection from the patch or striosomal compartment to the pars compacta is reciprocated by a projection from particular subgroups of dopaminergic neurons, which in the rat reside in the ventral tier of the pars compacta or in the ventrolateral part of the pars reticulata, and in the cat in the densocellular portion of the pars compacta (Gerfen et al., 1985, 1987; Jiméncz-Castellanos and Graybiel, 1987; see also Feigenbaum-Langer and Graybiel, 1989). Neurons in the dorsal strata of the pars compacta, in the ventral tegmental area, and in the retrorubral area project to the striatal matrix compartment. The striatal inputs to the latter parts of the mesencephalon originate in the ventral striatum (see above). At present it is unknown whether the ventral striatomesencephalic projections bear any relationship to the previously described neurochemical and cytoarchitectural compartments of the ventral striatum (Herkenham et al., 1984; Voorn et al., 1986, 1989; Groenewegen et al., 1989, 1991). Since most cortical and thalamic afferents of the ventral striatum respect compartmental boundaries (Groenewegen et al., 1987; Berendse et al., 1988, 1992; Berendse and Groenewegen, 1990), a compartmental ordering of ventral striatal efferents could enable particular combinations of inputs to interact with neurons projecting to distinct targets in the ventral mesencephalon, as is the case in the dorsal striatum (Gerfen, 1984, 1985, 1989; Jiménez-Castellanos and Graybiel, 1989).

Recently, it has been demonstrated that the mediolateral organization in the efferent connections of the nucleus accumbens (Nauta et al., 1978; Troiano and Siegel, 1978; Groenewegen and Russchen, 1984; Haber et al., 1990; Zahm and Heimer, 1990) is a reflection of the differential projection patterns of the neurochemically identifiable shell and core regions of this nucleus (Heimer et al., 1991). In a series of experiments performed in the course of a more extensive investigation of the organization of ventral striatal connections (Groenewegen et al., 1989, 1991), the anterograde tracer Phaseolus vulgaris leucoagglutinin (PHAL) was injected in various parts of the ventral striatum. In cases with apparently similarly placed striatal deposits, two different termination patterns could be observed in the mesencephalon. Labeled fibers either were largely confined to the pars reticulata of the substantia nigra or involved the ventral tegmental area, the pars compacta of the substantia nigra, and the retrorubral field. In the latter cases labeled fibers could also be traced caudally into the peribrachial region. These observations cannot be explained by core-shell differences and may therefore reflect the existence of discrete subpopulations of neurons related to the more detailed features of the neurochemical compartmentation of the ventral striatum (Voorn et al., 1989).

In order to label the ventral striatal neurons projecting to the different mesencephalic targets, retrograde tracer injections were made in the different parts of the dorsomedial substantia nigra, in the ventral tegmental area, and in the retrorubral field. Additional injections were placed in the peribrachial region to identify the neuronal population projecting to more caudal parts of the midbrain. The opioid peptide enkephalin (ENK), visualized immunohistochemically, was used as a marker for the ventral striatal compartments (Berendse et al., 1988; Voorn et al., 1989; Berendse and Groenewegen, 1990). A preliminary set of experiments, using fluorogold and diamidine yellow as retrograde tracers, provided us with the first indications of differential compartmental origins of the ventral striatomesencephalic projections (Groenewegen et al., 1989). The retrograde tracer cholera toxin subunit $\mathrm{B}(\mathrm{CTb})$ proved to be more sensitive and produced a more extensive filling of the dendritic trees of the rather small striatal projection neurons. Therefore, the present account is based on CTb experiments only. The analysis of a limited number of PHA-L injections in the nucleus accumbens and the ventral part of the caudate-putamen was necessary to supplement the data obtained by means of retrograde tracing.

\section{Materials and Methods}

In the present study, 32 female Wistar rats (Harlan/CPB, 7eist, The Netherlands) weighing 180-220 gm were used. Following anesthesia with an intramuscular injection of a mixture $(4: 3)$ of ketamine and $2-$ (2,6-xylidine)5,6-dihydro-1,3-thiazine (Rompun $\left.{ }^{\circledR}\right)$, in a dosage of $1 \mathrm{ml} /$ $\mathrm{kg}$, the animals were mounted in a stereotaxic apparatus. Via small burr holes in the exposed skull, retrograde or anterograde tracers were injected at the appropriate sites in the brain, using coordinales derived from the atlas of Paxinos and Watson (1986). Injections of the retrograde tracer CTb were placed in the ventral mesencephalon of 27 rats. The anterograde tracer PHA-L was injected into the ventral striatum of five rats.

Cholera toxin subunit B. A $1 \%$ solution of CTb (Sigma; Ericson and Blomqvist, 1988), containing $0.25 \mathrm{M}$ Tris, $1 \mathrm{M} \mathrm{NaCl}, 0.005 \mathrm{M} \mathrm{Na}_{2}$ EDTA,

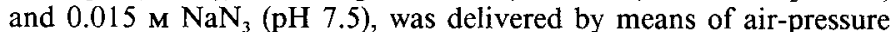
injections through glass micropipettes (internal tip diameter, $7-10 \mu \mathrm{m}$ ). Some of the earlier injections were placed with a $10 \mu \mathrm{l}$ Hamilton syringe. Using air-pressure pulses, 5-25 $\mathrm{nl}$ of the solution containing the tracer were injected over a period of 5-10 min. Deposits made with a Hamilton syringe were in the order of 100-200 nl. Following a 7-13 d postoperative period, the animals were deeply anesthetized with pentobarbital and perfused transcardially with $150 \mathrm{ml}$ of saline and $500 \mathrm{ml}$ of a fixative containing $4 \%$ paraformaldehyde and $15 \%$ picric acid in $0.1 \mathrm{M}$ phosphate

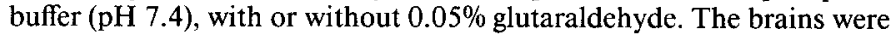
removed from the skull, postfixed for $\mathrm{l}-2 \mathrm{hr}$ in the perfusion fixative, and stored for $18-48 \mathrm{hr}$ at $4^{\circ} \mathrm{C}$ in a mixture of $20 \%$ glycerol and $2 \%$ dimethyl sulfoxide in distilled water. Sections were cut at $35 \mu \mathrm{m}$ on a freezing microtome and collected in $0.05 \mathrm{M}$ Tris-buffered saline $(\mathrm{pH}$ 7.6). Every second section was incubated for $18-48 \mathrm{hr}$ in goat anti-CTb antiserum (1:10,000; I ist Biochemicals), followed by successive $45 \mathrm{~min}$ incubations in donkey anti-goat whole serum (1:50; Nordic Immunology, Tilburg, The Netherlands) and goat peroxidase-antiperoxidase (PAP; 1:1500; Nordic Immunology). The goat anti-CTb antiserum was preincubated for $18 \mathrm{hr}$ with rat brain tissue to reduce background staining. All incubations and intervening rinses were in $0.05 \mathrm{M}$ Tris-buffered saline ( $\mathrm{pH} 7.6$ ) with $0.5 \%$ Triton X-100 (TBS-T). Following the last incubation, the sections were rinsed twice in TBS-T and twice in $0.1 \mathrm{M}$ phosphate buffer ( $\mathrm{pH} 7.4$ ). Thereafter, they were treated with Ni-enhanced diaminobenzidine (DAB; $12.5 \mathrm{mg}$ in $25 \mathrm{ml}$ of $0.1 \mathrm{M}$ phosphate buffer, $\mathrm{pH} 7.4$, with $1 \mathrm{ml}$ of $1 \%$ ammonium Ni-sulfate). Finally, the sections were rinsed, mounted from $0.2 \%$ gelatin, air dried, and coverslipped from xylene. One series of sections was counterstained with cresyl violet prior to coverslipping. Due to the presence of granular reaction product in the striatum, presumably caused by anterograde transport of $\mathrm{CTb}$, the distribution of ENK-immunoreactivity (ENK-IR) could not be visualized reliably in double-stained sections. Therefore, 
a series of adjacent sections was processed to visualize ENK as described below. The localization of the injection sites in the peribrachial region relative to the dopaminergic neurons of the A8 cell group was determined by comparison with a series of adjacent sections stained for tyrosine hydroxylase (see below).

Phaseolus vulgaris leucoagglutinin. The PHA-L tract-tracing method (Gerfen and Sawchenko, 1984) was used to trace the efferents to the mesencephalon of single compartments in the ventral striatum. Small deposits of PHA-L (Vector Laboratories) were delivered iontophoretically through glass micropipettes (external tip diameter, $10 \mu \mathrm{m}$ ) using a positive-pulsed $7.5 \mu \mathrm{A}$ current ( $7 \mathrm{sec}$ on, $7 \mathrm{sec}$ off; CCS-3 current source) for 10-15 min. The animals were allowed to survive for $7 \mathrm{~d}$, after which the animals were deeply anesthetized with pentobarbital and perfused transcardially with $100 \mathrm{ml}$ saline, followed by $500 \mathrm{ml}$ of a fixative containing $4 \%$ paraformaldehyde, $15 \%$ picric acid, and $0.05 \%$

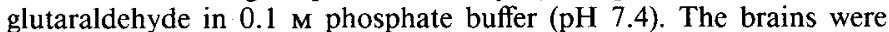
removed from the skull, stored in the same fixative for $1-2 \mathrm{hr}$, and then transferred to a mixture of $20 \%$ glycerol and $2 \%$ dimethyl sulfoxide in distilled water for overnight storage at $4^{\circ} \mathrm{C}$. Subsequently, the brains were cut on a freezing microtome at $35 \mu \mathrm{m}$ and the sections were collected in $0.05 \mathrm{M}$ Tris-buffered saline ( $\mathrm{pH} 7.6)$.

Two series of sections were stained for PHA-L using the peroxidaseantiperoxidase method (Sternberger, 1979) according to the protocol described previously (Berendse and Groenewegen, 1990; Berendse et al., 1992). One of the PHA-L-stained series was counterstained with cresyl violet before coverslipping. In order to determine the compartmental localization of the tracer injection, a series of adjacent sections through the striatum was processed to visualize the distribution of ENK as described below. The distribution of PHA-L-labeled fibers and terminals in the midbrain was charted with the aid of a drawing tube on a standard Zeiss microscope.

Enkephalin immunohistochemistry. Sections were incubated in rabbit anti-leu-enkephalin antiserum (1:4000; UCB-Bioproducts, Braîne L'Alleud, Belgium) for $18 \mathrm{hr}$ at $4^{\circ} \mathrm{C}$, followed by swine anti-rabbit whole serum (1:50; Nordic Immunology) and then by rabbit-PAP (1:1500; Dakopatts, Glostrup, Denmark) each for $45 \mathrm{~min}$ at $20^{\circ} \mathrm{C}$. All incubations and intervening rinses were in TBS-T. DAB-Ni (see CTb protocol above) was used as a chromogen.

Tyrosine hydroxylase immunohistochemistry. In experiments with CTb injections in the peribrachial area, tyrosine hydroxylase immunoreactivity (mouse anti-tyrosine hydroxylase, 1:3000; Incstar, Stillwater, MN) was demonstrated with a similar protocol as described above for ENKIR.

\section{Results}

Distribution of ventral striatal neurons projecting to the mesencephalon

The cells of origin of the ventral striatal projections to the mesencephalon were determined by placing deposits of CTb at several loci in the midbrain known to receive ventral striatal efferents. Seven CTb deposits were confined to the ventral tegmental area. In six cases, the deposits were restricted to the medial part of the substantia nigra, whereas in another six cases they additionally involved the ventral tegmental area. The remainder of the CTb injections $(n=8)$ were placed more caudally in the mesencephalon. All deposits resulted in the retrograde labeling of neurons in the ipsilateral striatum only, except for those cases in which the deposit in the ventral tegmental area extended across the midline, following which bilateral labeling was observed.

Ventral tegmental area. In case RC-89059, a large injection of $\mathrm{CTb}$ was placed in the ventral tegmental area. The deposit virtually fills the area and extends only minimally into the adjacent medial part of the substantia nigra (Fig. $1 A$ ). The retrogradely labeled cells are confined to the nucleus accumbens and the caudoventral part of the caudate-putamen or fundus striati. Most of the neurons occur in the medial and ventral parts of the shell region of the nucleus accumbens (see Fig. $4 A$ ). Smaller numbers of labeled neurons are found in the rostral part of the nucleus accumbens, mostly in its medial half (Fig. 1C). Caudal to the nucleus accumbens, retrogradely labeled neurons reside in the area surrounding the posterior limb of the anterior commissure and in the medial part of the fundus striati.

More restricted CTb deposits (cases RC-90046 and RC-90404), which produce complementary patterns of retrograde labeling, demonstrate that a certain degree of topography is present in the projections from the ventral striatum to the ventral tegmental area. The deposit in case RC-90046 involves only the lateral part of the ventral tegmental area, adjacent to the medial terminal nucleus of the accessory optic tract that shields the area from the adjacent medial part of the substantia nigra pars compacta (Figs. $1 B, 2$ ). More caudally, where this nucleus is absent, the periphery of the injection site may involve the pars compacta of the substantia nigra. As in case RC-89059, the shell region of the nucleus accumbens is most heavily labeled (Figs. 1E; $2 B, C)$. However, the dorsomedial part of the shell region is now virtually devoid of labeled neurons (Fig. 2C). Furthermore, in the lateral part of the nucleus accumbens and the ventrolateral part of the caudate-putamen, several groups of labeled neurons are present (Fig. $2 A-D$ ). Caudal to the nucleus accumbens, the retrogradely labeled neurons have the same distribution as in case RC-89059 (Fig. 2D,E). In case RC-90404, the CTb deposit involves the ventromedial part of the ventral tegmental area and the rostrodorsal part of the interpeduncular nucleus (Fig. 3 ). Retrogradely labeled neurons are concentrated in the dorsomedial part of the shell region of the nucleus accumbens (Fig. $3 A-C)$. Only few neurons reside in the ventromedial part of the shell region and in the fundus striati (Fig. $3 D, E$ ).

$\mathrm{CTb}$ injections in the lateral part of the ventral tegmental area that clearly involve the medial part of the substantia nigra produce additional retrograde labeling in the rostromedial part of the caudate-putamen and the central or core part of the nucleus accumbens. Furthermore, retrogradely labeled neurons in the rostral part of the nucleus accumbens are more numerous than in cases in which the injection site is confined to the ventral tegmental area.

The distribution of the retrogradely labeled neurons in the ventral striatum following injections of CTb in the ventral tegmental area is not homogeneous. Labeled neurons in the rostrolateral part of the nucleus accumbens are located outside of the large areas of high ENK-IR that surround the anterior commissure, as can be appreciated when PHA-I-stained sections are compared with adjacent sections stained for ENK (Fig. 1C,D). In the medial part of the shell region of the nucleus accumbens, several circumscribed areas are devoid of retrogradely labeled neurons (Fig. 4A). Comparison with adjacent sections stained for ENK reveals that immunoreactivity for ENK is virtually absent from these areas (Fig. $4 A, B$ ). In cresyl violet-counterstained sections, it can be observed that the unlabeled areas coincide with clusters of densely packed cells (Fig. 4C). As illustrated in Figure $4 D$, the dendrites of the retrogradely labeled neurons surrounding these areas appear to avoid entering them. In the ventral part of the shell region, the situation is more complex. Like in the medial part of the shell, the retrogradely labeled neurons do not overlie clusters of densely packed cells. In addition, the labeled neurons are heterogeneously distributed over the areas surrounding the cell clusters (Fig. $1 E, F$ ). Rostrally, they are concentrated in two strands of neurons, one adjacent to the core and the other close to the outer border of the shell. At several points the two strands are interconnected. Caudally, the retrogradely labeled neurons are arranged in groups that are interposed between the striatal cell bridges. The neurons 

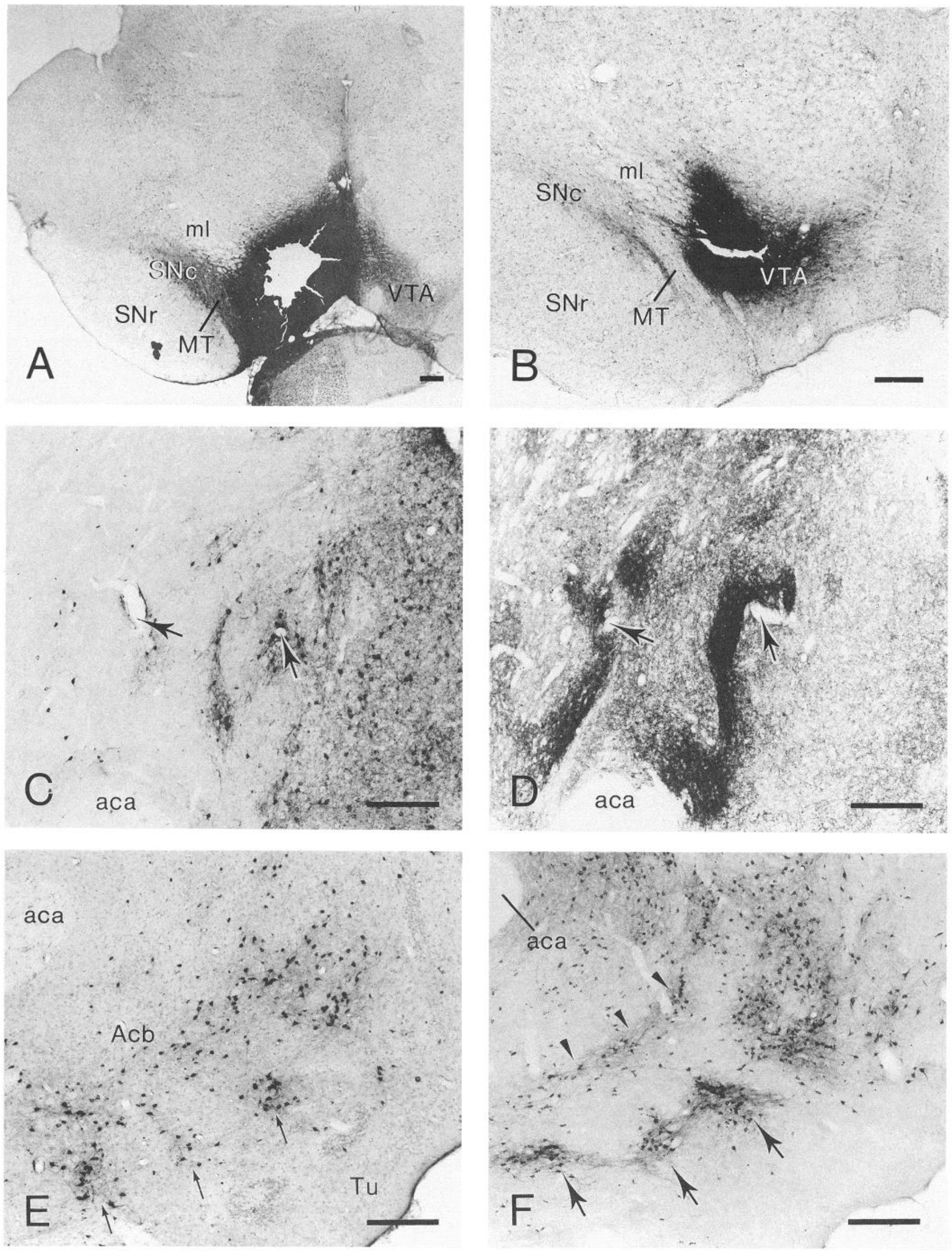
in the cell bridges and morphologically similar neurons in the ventral part of the shell are not retrogradely labeled (Fig. $1 E$ ). In the fundus striati, retrogradely labeled neurons are absent from the cell clusters that occasionally can be observed in this area.

Substantia nigra. In case RC-89631, an injection of CTb was placed in the medial part of the substantia nigra, primarily involving the caudal part of the pars compacta but extending rostrally into the pars reticulata (Fig. 5). In the striatum, the majority of the retrogradely labeled neurons are located in the rostromedial part of the caudate-putamen and in the adjacent dorsal part of the nucleus accumbens (Fig. $5 A, B$ ). Smaller numbers of labeled neurons are present in caudomedial parts of the caudate-putamen and in more central parts of the nucleus accumbens (Fig. 5C-E). The shell region of the nucleus accumbens contains only a few faintly labeled neurons (Fig. $5 B$ ). Although neurons in some parts of the caudate-putamen tend to be arranged in clusters (Fig. $5 B, C$ ), in other areas the distribution is more homogeneous (Fig. $5 A$ ).

In other cases, attempts were made to restrict retrograde tracer deposits either to the pars compacta or to the pars reticulata of the medial substantia nigra. The CTb deposit in case RC-90328 is centered in the medial one-third of the pars compacta, and, although the involvement of the pars reticulata is minimal, it unavoidably includes the lateral part of the ventral tegmental area (Fig. 6A). Numerous clusters of retrogradely labeled neurons occupy the rostral and medial parts of the caudate-putamen (Fig. 6C). A few labeled neurons are scattered between the clusters. Slightly larger aggregates of labeled neurons occur in the rostrolateral part of the nucleus accumbens (Fig. $6 C, D$ ). In caudal parts of the nucleus, the neurons are more diffusely distributed. Retrogradely labeled neurons are also present ventromedially in the shell of the nucleus accumbens and in the fundus striati. A comparison of the distribution of the retrogradely labeled neurons with the distribution of ENK-IR in adjacent sections demonstrates that the aggregates of labeled neurons in the rostrolateral part of the nucleus accumbens are interposed between the large areas of strong ENK-IR (Fig. 6D,E). More caudally, in the border region between the ventral part of the caudate-putamen and the nucleus accumbens, small clusters of neurons, retrogradely labeled following injections in the substantia nigra pars compacta, overlie patches of strong ENK-IR (Fig. $7 A / A^{\prime}, B / B$ ).

An injection centered in the dorsomedial part of the substantia nigra pars reticulata with additional involvement of the ventral part of the pars compacta (case RC-90329; Fig. 6B) results in retrograde labeling of neurons in ventral parts of the caudateputamen and in the nucleus accumbens. The shell region of the latter nucleus is not labeled. Probably as a result of the involvement of the pars compacta, the distribution of the retrogradely labeled neurons is rather homogeneous.

In case RC-89058, a large CTb deposit involves the caudal part of the pars reticulata and, at more rostral levels, spreads into the medial part of the pars compacta. Retrogradely labeled neurons are widely distributed over the striatum. Nevertheless, a comparison of adjacent sections through the rostrolateral part of the nucleus accumbens stained for ENK and CTb demonstrates that the majority of the labeled neurons are concentrated in the large areas of strong ENK-IR surrounding the anterior commissure (Fig. $7 \mathrm{C} / \mathrm{C}$ ).

Retrorubral field. Retrograde tracer deposits in various parts of the retrorubral field $(n=4)$ resulted in similar distribution patterns of retrogradely labeled neurons. The deposit in case $\mathrm{RC}-90156$ is centered in the caudal part of the retrorubral field (Figs. $8 A, 9$ ). In the striatum, retrogradely labeled neurons are confined to the nucleus accumbens and the ventral part of the caudate-putamen (Fig. 9). The majority of the neurons reside in the ventromedial part of the shell region of the nucleus accumbens, in the area surrounding the posterior limb of the anterior commissure, and in the medial part of the fundus striati (Fig. 9C-E). Smaller numbers of labeled neurons are present dorsomedially in the shell region and in the rostral part of the nucleus accumbens, predominantly in the vicinity of the anterior commissure (Fig. 9A-C). A few small groups of neurons reside in the ventrolateral part of the caudate-putamen (Fig. 9B-D). Scattered neurons are found in the lateral part of the fundus striati (Fig. 9D,E).

The retrogradely labeled neurons in the ventromedial part of the shell region of the nucleus accumbens are concentrated in groups at the outer border of the shell, much like the neurons labeled following injections in the ventral tegmental area (Fig. $9 C)$. They are located outside of the cell clusters. In cresyl violetcounterstained sections, groups of labeled neurons alternate with strands of cells that extend into the striatal cell bridges (Fig. $10 A, B)$. In the rostrolateral part of the nucleus accumbens, the majority of the labeled neurons surrounding the anterior commissure are located outside of the ENK-positive areas. However, a distinct number of neurons are found in an area of strong ENK-IR adjacent to the external capsule. Most of the small groups of neurons in the ventrolateral part of the caudate-pu-

\footnotetext{
Figure 1. $A$ and $B$, Photomicrographs of CTb deposits in the ventral tegmental area (VTA). The large deposit in $A$ (RC-89059) completely fills the ventral tegmental area and may have encroached upon the adjacent medial part of the substantia nigra. The much smaller injection site in $B$ (shown at a higher magnification; RC-90046) involves only the lateral part of the ventral tegmental area and is shielded from the substantia nigra pars compacta $(S N C)$ by the medial terminal nucleus of the accessory optic tract $(M T)$. $C$ and $D$, Pair of adjacent transverse sections through the rostrolateral part of the nucleus accumbens following the injection of CTb illustrated in $A$ to show the relationship of retrogradely labeled neurons $(C)$ with the distribution of ENK-IR $(D)$. The small groups of labeled neurons that surround the anterior commissure are located outside of the large ENK-positive areas. Arrows mark corresponding blood vessels. Note that the majority of the retrogradely labeled neurons in $C$ is present in the medial part of the nucleus accumbens. $E$ and $F$, Photomicrographs illustrating the heterogeneous distribution of $C T$-labeled neurons in the ventromedial part of the shell region of the nucleus accumbens $(A c b)$ following injections in the ventral tegmental area. The section in $E$ was counterstained with cresyl violet and was taken from case RC-90046 $(B)$. Note that the labeled neurons at the ventral border of the nucleus accumbens are arranged in groups (arrows in $E$ ) that are interposed between the striatal cell bridges connecting the nucleus accumbens with the olfactory tubercle $(T u)$. The cells that lie in direct continuity with the cell bridges are unlabeled. The section in $F$ was taken from a case with a large injection of $\mathrm{CTb}$ in the ventral tegmental area that extends into the medial part of the substantia nigra. In this section, it can be better appreciated that the labeled neurons in the shell region are arranged in two bands, one adjacent to the core region (arrowheads) and the other to the ventral border of the nucleus accumbens (arrows). Note that the latter consists of separate cell groups (see also $E$ ). aca, anterior commissure, anterior part; $m l$, medial lemniscus; $S N r$, substantia nigra, pars reticulata. Scale bars, $250 \mu \mathrm{m}$.
} 


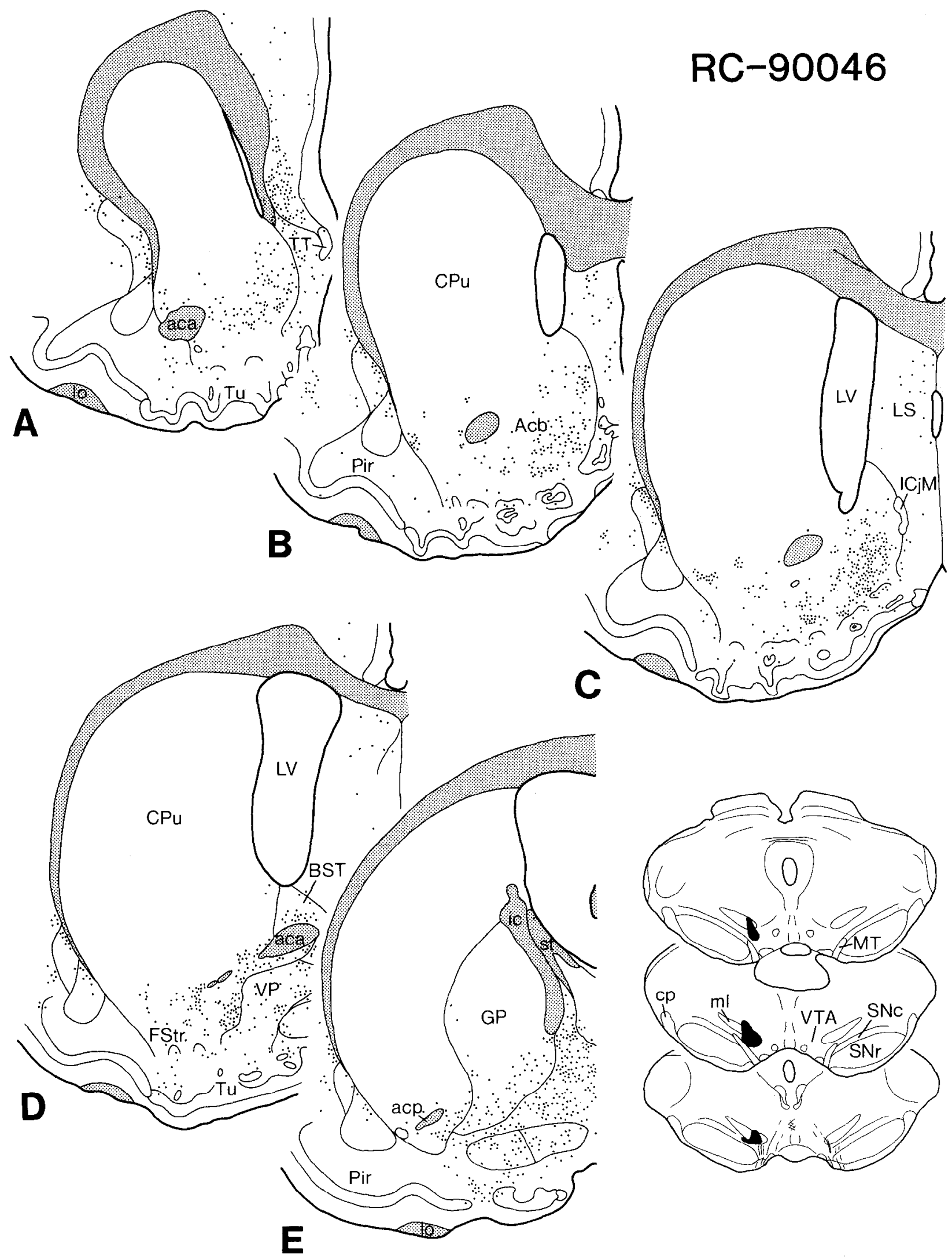

Figure 2. Chartings of retrogradely labeled neurons in five frontal sections through the striatum following an injection of CTb in the lateral part of the ventral tegmental area (VTA; RC-90046). The injection site is drawn in the lower right corner and is shown photographically in Figure $1 B$. Each $d o t$ represents a single labeled cell. $a c a$, anterior commissure, anterior part; $a c p$, anterior commissure, posterior part; $A c b$, nucleus accumbens; $B S T$, bed nucleus of the stria terminalis; $c p$, cerebral peduncle; $C P u$, caudate-putamen; FStr, fundus striati; $G P$. globus pallidus; $i c$, internal capsule; $I C j M$, major island of Calleja; $l o$, lateral olfactory tract; $L S$, lateral septum; $L V$, lateral ventricle; $m l$, medial lemniscus; $M T$, medial terminal nucleus of the accessory optic tract; Pir, piriform cortex; $S N c$, substantia nigra, pars compacta; $S N r$, substantia nigra, pars reticulata; st, stria terminalis; $T T$, taenia tecta; $T u$, olfactory tubercle; $V P$, ventral pallidum. 


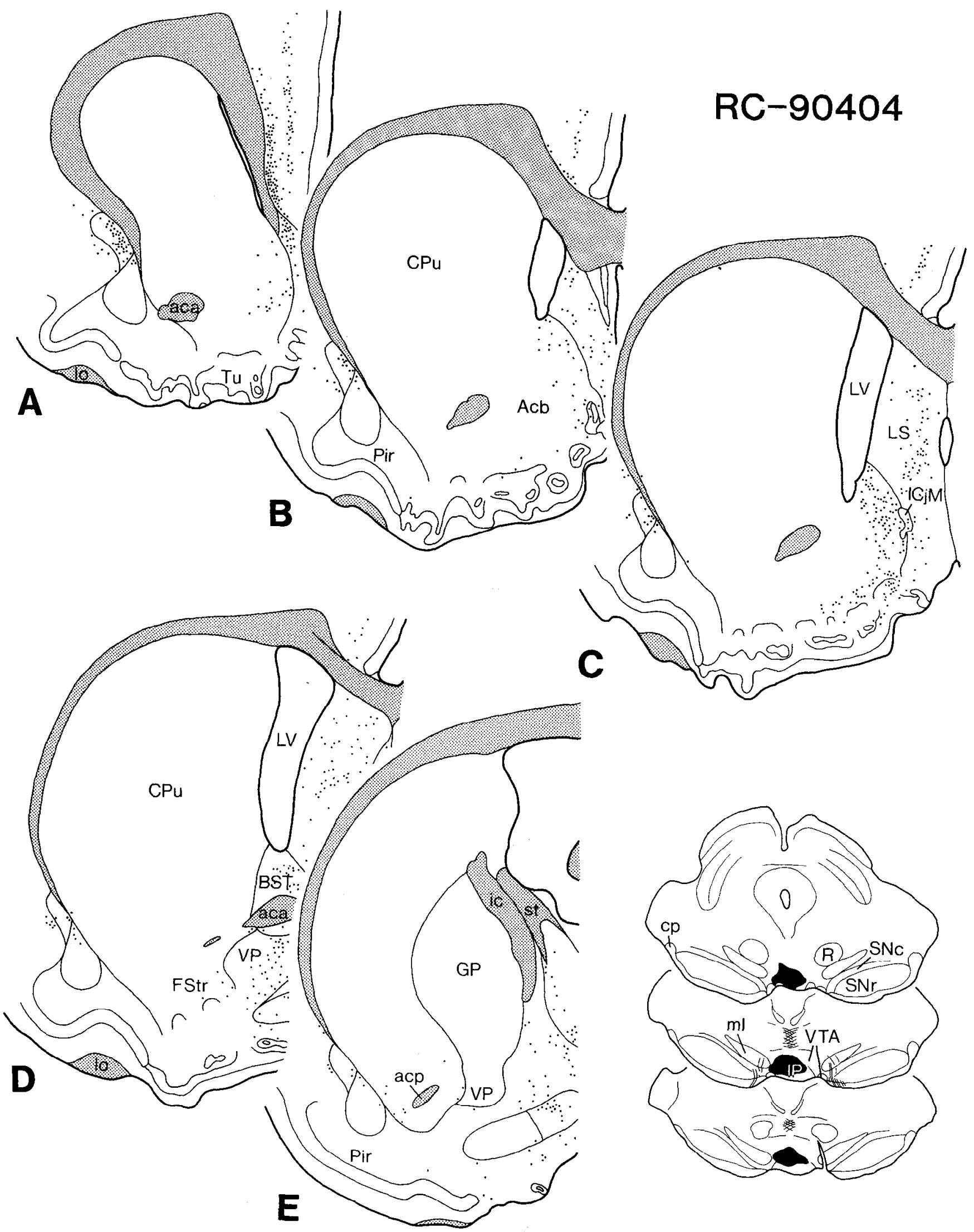

Figure 3. Chartings of retrogradely labeled neurons in five frontal sections through the striatum resulting from an injection of $\mathrm{CTb}$ involving the ventromedial part of the ventral tegmental area (RC-90404). The injection site is drawn in the lower right corner. Each dot represents a single labeled cell. $I P$, interpeduncular nucleus; $R$, red nucleus; for other abbreviations see Figure 2. 

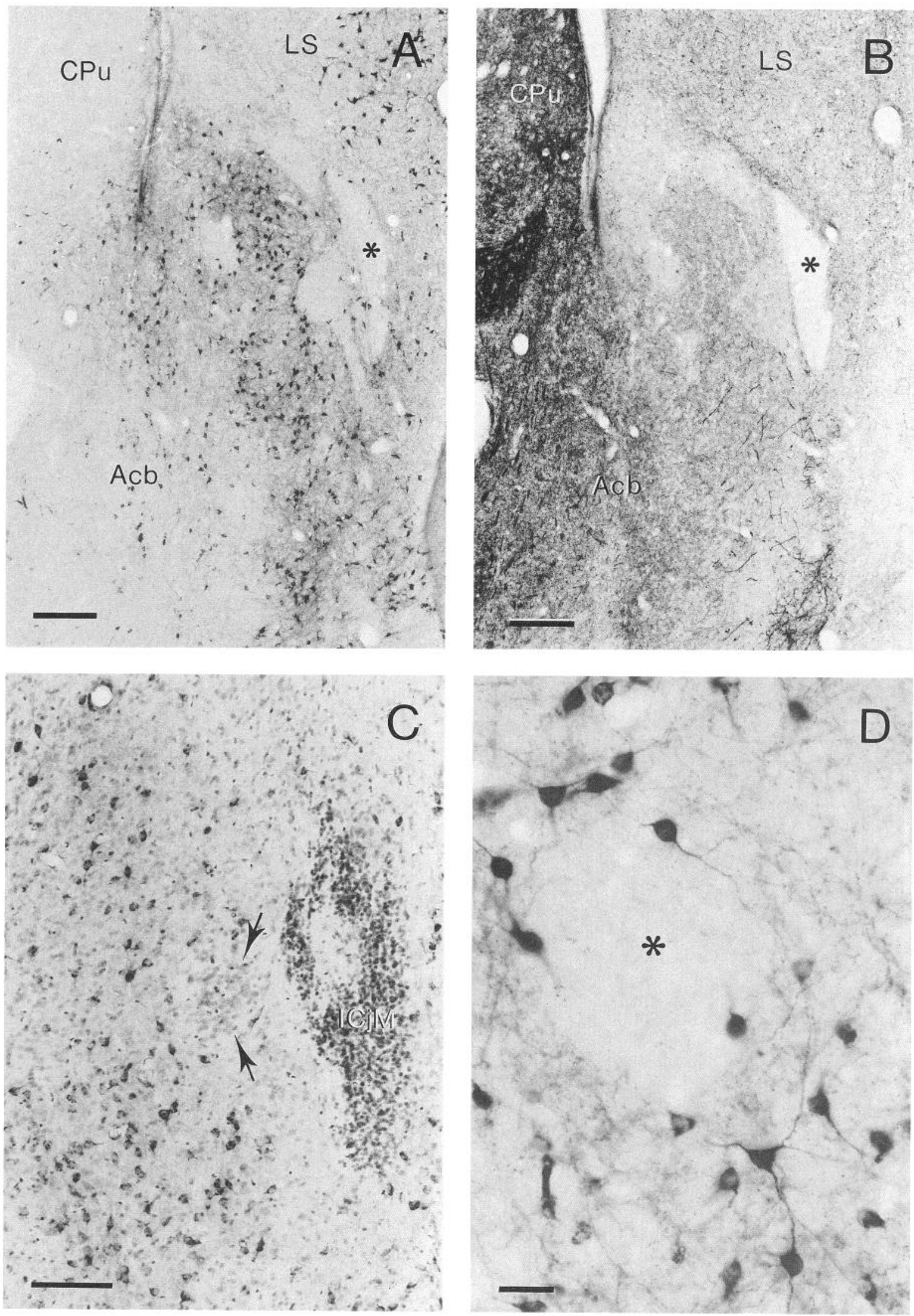

Figure 4. $A$ and $B$, Pair of adjacent transverse sections through the caudomedial part of the nucleus accumbens $(A c b)$ to show the relationship of retrogradely labeled neurons $(A)$ with the distribution of ENK-IR $(B)$ following an injection of CTb in the ventral tegmental area. Note that labeled neurons are absent from several areas where ENK-IR is very weak. One of these areas lies close to the major island of Calleja (asterisks). See also $D$. $C$, Photomicrograph of the caudomedial part of the nucleus accumbens at the level of the major island of Calleja (ICjM) immunostained for $\mathrm{CTb}$ and counterstained with cresyl violet following an injection centered in the lateral part of the ventral tegmental area. Note that the cell cluster immediately lateral to the major island of Calleja (arrows) is devoid of CTb labeled neurons. D, High-power photomicrograph of CTb labeled neurons surrounding an area of high cell density and weak ENK-IR marked with an asterisk (see $A-C$ ). Note that the dendrites of the neurons do not enter the area but rather seem to encircle it. $C P u$, caudate-putamen; $L S$, lateral septum. Scale bars: $A$ and $B, 200 \mu \mathrm{m} ; C, 100 \mu \mathrm{m} ; D, 25 \mu \mathrm{m}$. 


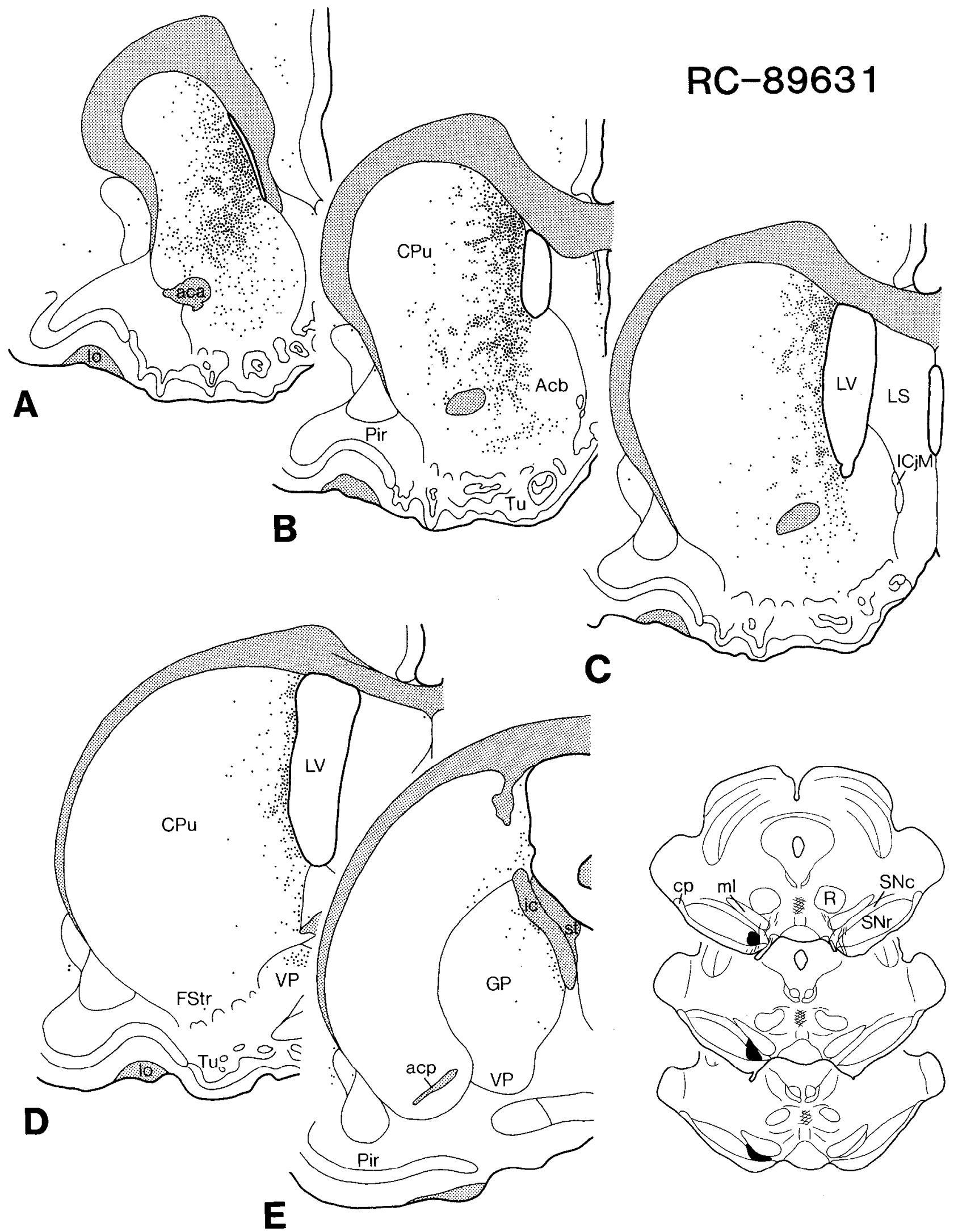

Figure 5. Chartings of retrogradely labeled neurons in five frontal sections through the striatum that result from an injection of CTb in the medial part of the substantia nigra (RC-89631). The injection site is drawn in the lower right corner. Note the clustering of labeled neurons in the medial part of the caudate-putamen ( $B$ and $C$ ). Each dot represents a single labeled cell. $R$, red nucleus; for other abbreviations, see Figure 2. 

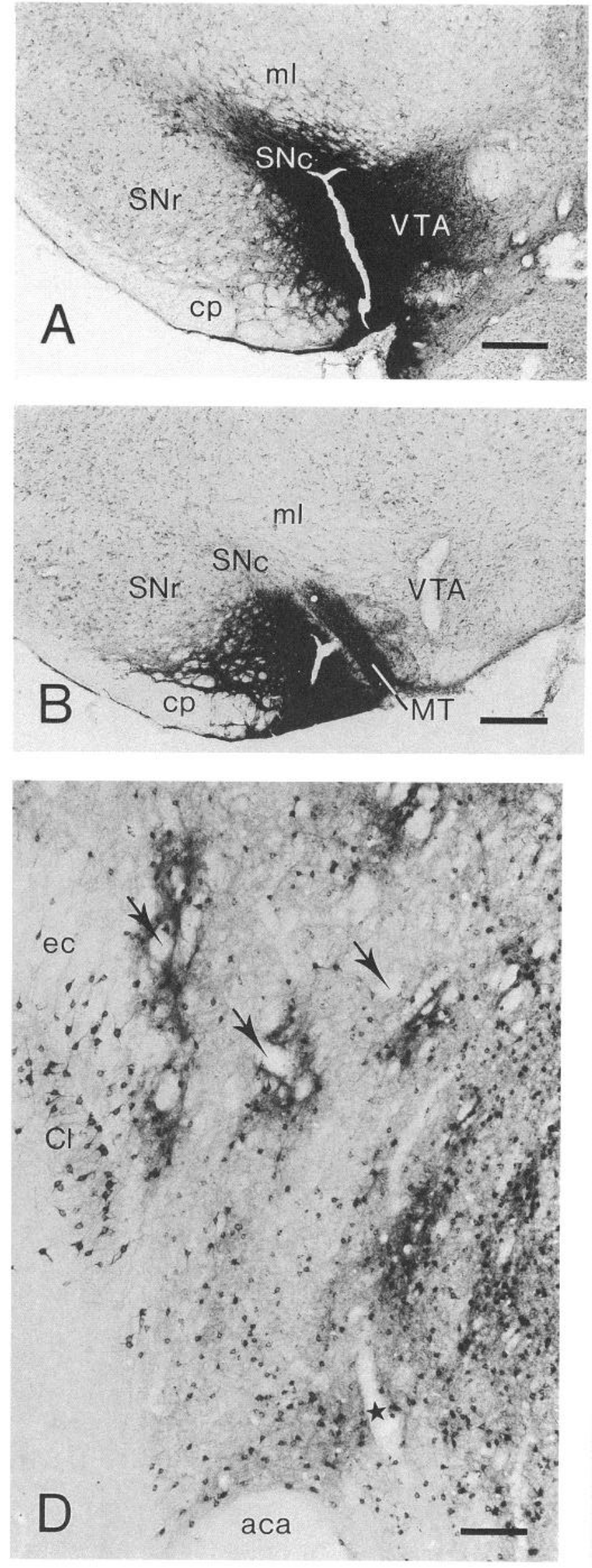
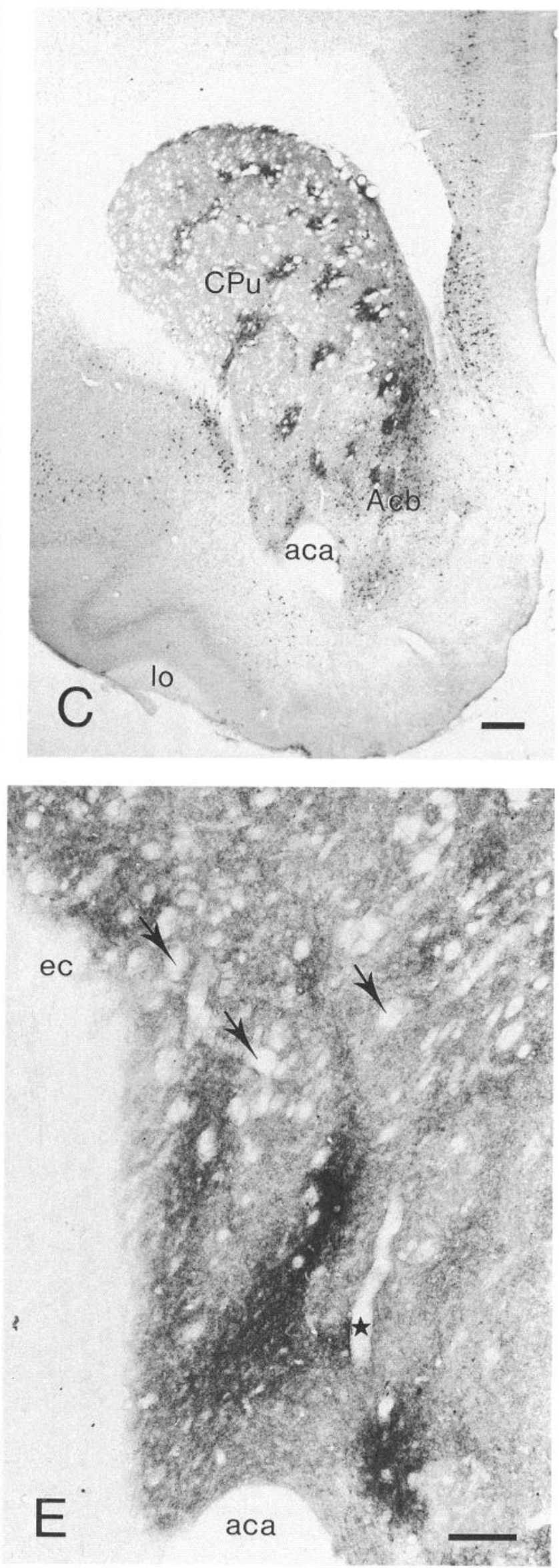
tamen coincide with patches of strong ENK-IR, but in all experiments a single group of retrogradely labeled cells was found to overlap an area of very low ENK-IR (Fig. 10C,D).

Peribrachial region. Several CTb injections were made in the peribrachial region $(n=4)$. To assess a possible involvement of the A8 dopaminergic cell group in the injection site, a series of adjacent sections was stained for tyrosine hydroxylase. In case $\mathrm{KC}-90279$, the $\mathrm{Cl}$ ' b deposit was placed in the peribrachial region caudal to the $A 8$ cell group and ventral to the cuneiform nuclcus, the ventral part of which is included in the injection site (Figs. $8 B, 11$ ). Caudally, the deposit extends into the parabrachial nuclei. Separate from the rest of the deposit, some reaction product was observed in the ventromedial part of the central gray substance. As illustrated in Figure 11, the distribution of retrogradely labeled neurons in the striatum resembles that observed after injections in the retrorubral field, but in case RC-90279 the majority of the labeled neurons are concentrated in caudal parts of the striatum. Most densely labeled are the ventromedial part of the shell region of the nucleus accumbens, the medial part of the fundus striati, and the area surrounding the posterior limb of the anterior commissure (Fig. $11 C-E$ ). Far fewer retrogradely labeled cells are present in rostral parts of the nucleus accumbens and in the ventrolateral part of the caudate-putamen (Fig. $\perp \perp A-D$ ). In the latter area, the neurons are arranged in clusters (Fig. $11 B-D$ ). The compartmental relationships of the neurons that project to the peribrachial region are similar to those of the neurons issuing fibers to the retrorubral field (see above).

\section{Anterograde tracing experiments}

In order to substantiate further the differential projection patterns in the ventral mesencephalon of neurons in different ventral striatal compartments, we made an attempt to inject the anterograde tracer PHA-L into single compartments. The injections were placed in the rostrolateral part of the nucleus accumbens $(n=3)$ and in the ventromedial part of the caudateputamen $(n=2)$. A series of adjacent sections stained for ENK was used to determine the position of each PHA-L deposit in relation to the compartments. In two cases with injection sites in the rostrolateral part of the nucleus accumbens, the deposits are largely restricted to a single compartment. In case RP-90455, the majority of the PH $\Lambda$-L-labeled neurons are located in the large areas of strong ENK-IR in the vicinity of the anterior commissure (Figs. 12A/A', 13A). It is remarkable that the deposit, consisting of the PHA-L-filled cell bodies and their dendrites, corresponds in shape with the compartment in which it is centered (Fig. 12B). As illustrated in Figure 13A, the great majority of the terminating PHA-L-labeled fibers in the mesencephalon occupy the dorsomedial part of the substantia nigra pars reticulata. Fewer fibers are present in the pars compacta and the caudolateral part of the pars reticulata, whereas sporadic fibers were observed in the rostral part of the retrorubral area. The PHA-L deposit in case RP-90480 is exactly interposed between the large areas of strong ENK-IR, and only few neurons are labeled inside these areas (Figs. $12 C / C^{\prime}, 13 B$ ). In the mesencephalon, most PHA-L-labeled fibers occupy the pars compacta of the substantia nigra, the lateral part of the ventral tegmental area, and the retrorubral field (Fig. 13B). However, especially at midrostrocaudal levels, $\mathrm{PH} \Lambda$-L-labeled fibers can be observed in the pars reticulata as well (Fig. 13B). The labeled fibers in the rostral part of the pars reticulata are predominantly of the passing type. PHA-L injections that were placed in the ventromedial part of the caudate-putamen could not be restricted to a single compartment.

\section{Discussion}

The principal finding of the present study is that the projections from the ventral striatum to the dopaminergic cell groups in the ventral mesencephalon and those to the substantia nigra pars reticulata originate from distinct subpopulations of neurons that respect neurochemically and cytoarchitectonically defined compartmental boundaries (Herkenham et al., 1984; Voorn et al., 1986, 1989), thus establishing an organization similar to that of the dorsal striatum (Gerfen, 1984, 1985; Gerfen et al., 1985; Jiménez-Castellanos and Graybiel, 1989). Since the afferents from the midline and intralaminar thalamic nuclei and from the prefrontal cortex to the ventral striatum are likewise compartmentally ordered (Herkenham et al., 1984; Berendse and Groenewegen, 1990; Berendse et al., 1992), the present results imply that specific sets of cortical and thalamic inputs may interact with neurons projecting to distinct targets in the mesencephalon. Recent observations indicating that the great majority of the ventral striatal neurons with projections to the ventral mesencephalon have a dendritic tree that is confined to the compartment in which the parent cell body resides (Groenewegen et al., 1990a; Arts and Groenewegen, 1992; cf. Herkenham et al., 1984; Penny et al., 1988; Kawaguchi et al., 1989) strengthen this assumption. Although both the dorsal striatum and the ventral striatum accommodate separate populations of neurons, largely located in different compartments, that project either to the dopaminergic cell groups in the ventral mesencephalon or to the substantia nigra pars reticulata (Gerfen, 1984, 1985; Gerfen et al., 1985; Jiménez-Castellanos and Graybiel, 1989; present results), the neurochemical characteristics and connections of the ventral striatal compartments appear to vary from one part of the ventral striatum to the other. In the caudodorsal part of the nucleus accumbens and the adjacent ventral

\footnotetext{
Figure 6. $A$ and $B$, Photomicrographs of CTb deposits in the medial part of the substantia nigra. $A$, Injection centered in the pars compacta of the substantia nigra $(S N C)$. and additionally involving the adjacent lateral part of the ventral tegmental area (VTA; RC-90328). The pars reticulata of the substantia nigra $(S N r)$ is largely spared. $B$, Injection in the pars reticulata of the substantia nigra with additional involvement of the ventral part of the pars compacta (RC-90329). $C$, Transverse section through the rostral part of the striatum illustrating the distribution of $C$ Tb-labeled neurons following the injection in $A$. Clusters of neurons are distributed throughout the caudate-putamen $(C P u)$ and the adjacent lateral and dorsal parts of the nucleus accumbens $(A c b)$. Only a few neurons are located outside the clusters, scattered throughout the striatum. Note that the groups of neurons in the lateral and ventral parts of the striatum are larger than the medially and dorsally located clusters. $D$ and $E$, Adjacent transverse sections through the rostrolateral part of the nucleus accumbens illustrating the relationship between the distribution of CTb-labeled neurons $(D)$ and that of ENK-IR $(E)$ following an injection centered in the medial part of the substantia nigra pars compacta $(A)$. Arrows in $D$ and $E$ indicate corresponding fiber bundles; stars mark the same blood vessel. Note that the majority of the neurons in $D$ are interposed between the large areas of strong ENK-IR (compare with Fig. $7 C / C^{\prime}$ ). $a c a$, anterior commissure, anterior part; $C l$, claustrum; $c p$, cerebral peduncle; $e c$, external capsule; $l o$, lateral olfactory tract; $m l$, medial lemniscus; $M T$, medial terminal nucleus of the accessory olfactory tract. Scale bars: $A-C, 350 \mu \mathrm{m} ; D$ and $E$, $150 \mu \mathrm{m}$.
} 


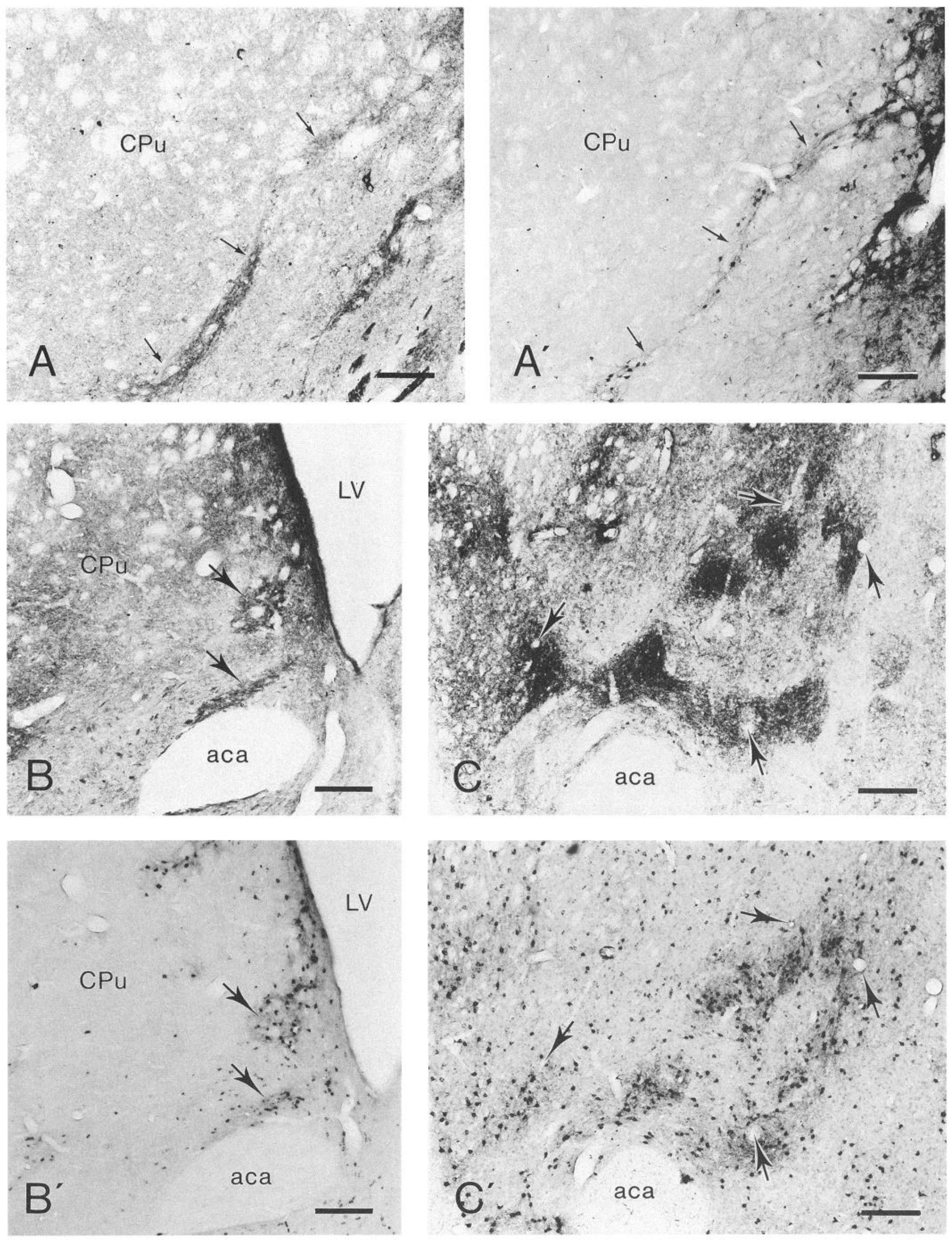



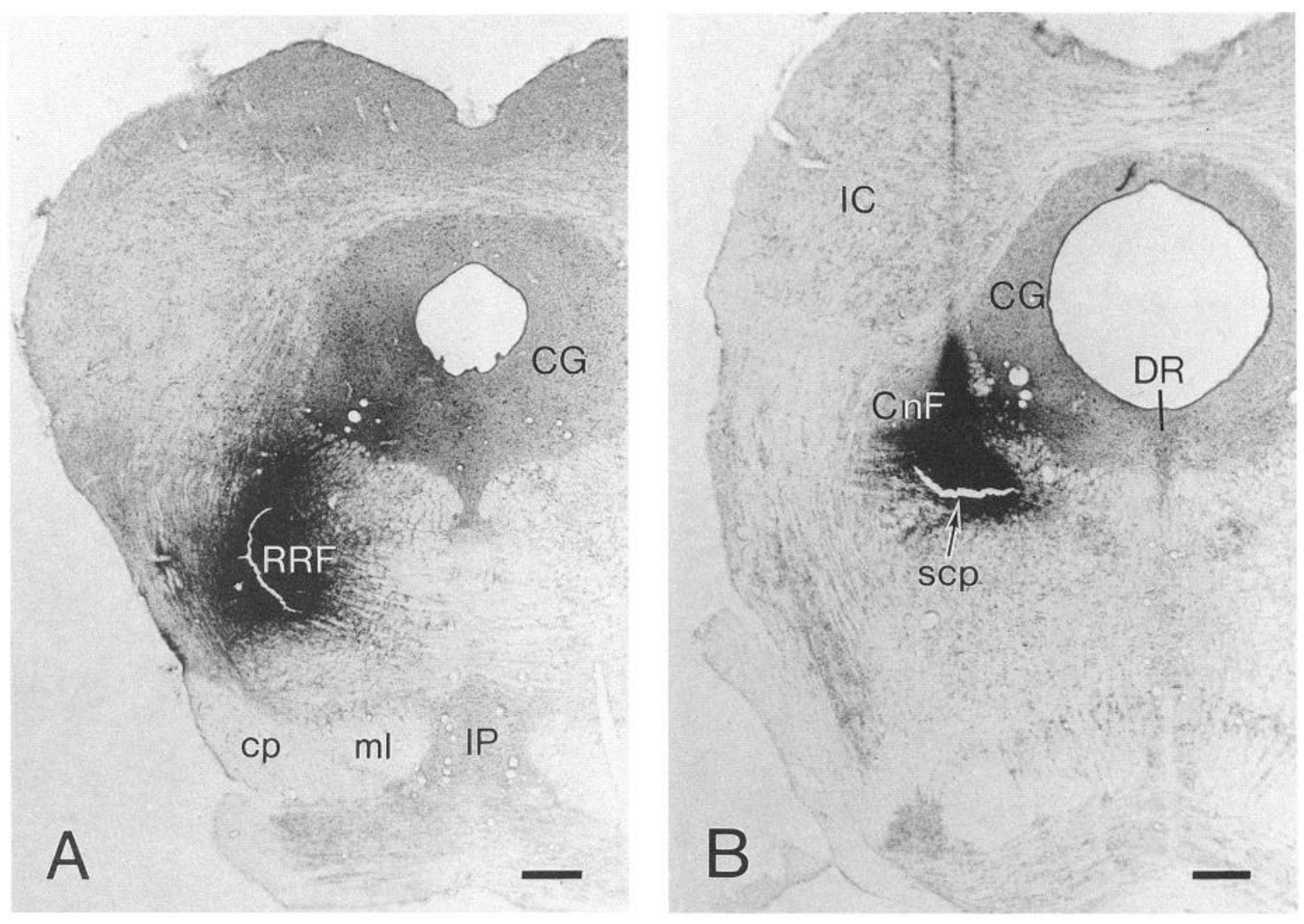

Figure 8. A, CTb deposit in the caudal part of the retrorubral field ( $R R F$; RC-90156). $B, \mathrm{CTb}$ injection site in the peribrachial region, centered just ventral to the cuneiform nucleus ( $C n F$; RC-90279). $C G$, central gray; $c p$, cerebral peduncle; $D R$, dorsal raphe nucleus; $I C$, inferior colliculus; $I P$, interpeduncular nucleus; $m l$, medial lemniscus; $s c p$, superior cerebellar peduncle. Scale bars, $400 \mu \mathrm{m}$.

part of the caudate-putamen, the compartments are comparable to those of the dorsal striatum, but in the shell and in the most rostrolateral part of the core of the nucleus accumbens, compartments with unique combinations of neurochemical characteristics and afferent and efferent relationships occur (Herkenham et al., 1984; Voorn et al., 1986, 1989; Berendse and Groenewegen, 1990; Berendse et al., 1992; present results; see also Martin et al., 1991). The anatomical heterogeneity of the ventral striatum may, at least in part, account for the divergent effects of lesions or pharmacological manipulations of this structure (e.g., Lorens et al., 1970; Pijnenburg and Van Rossum, 1973; Phillips et al., 1975; Smith and Holland, 1975; Evans and Vaccarino, 1986; Mogenson and Wu, 1988; Brutus et al., 1989).

\section{Origin of ventral striatal projections to the mesencephalon}

Ventral tegmental area. The present observations confirm that the projections from the striatum to the ventral tegmental area originate primarily in medial parts of the nucleus accumbens (Nauta et al., 1978; Troiano and Siegel, 1978; Phillipson, 1979c; Groenewegen and Russchen, 1984; Heimer et al., 1991), but also indicate that such projections are not limited to the shell region (cf. Heimer et al., 1991). A significant contribution stems from neurons in more rostral and lateral parts of the nucleus accumbens and in the area surrounding the posterior limb of the anterior commissure, including the medial part of the fundus striati. Consequently, neurons in the shell region of the nucleus accumbens as well as smaller groups of neurons in other parts of the ventral striatum may influence the activity of dopaminergic neurons in the ventral tegmental area that project to a large portion of the striatum (Fallon and Moore, 1978; Björklund and Lindvall, 1984; Gerfen et al., 1987). A certain degree of topography appears to be present in the projections from the ventral striatum to the ventral tegmental area. The medial part of this area receives its striatal input almost exclusively from the most

Figure 7. $A / A^{\prime}$ and $B / B^{\prime}$, Two pairs of adjacent transverse sections to show the relationship of CTb-labeled neurons $\left(A^{\prime}\right.$ and $\left.B\right)$ with the distribution of ENK-IR $(A$ and $B)$ in the ventromedial part of the caudate-putamen $(C P u)$ and the adjacent dorsal part of the nucleus accumbens following injections centered in the medial part of the substantia nigra pars compacta. CTb-labeled neurons in the ventromedial part of the caudate-putamen overlie a thin, elongated area of strong ENK-IR (arrows in $A$ and $A$ ). Note the exact correspondence in shape and position of the strand of neurons in $A^{\prime}$ and the ENK-positive area in $A . B / B^{\prime}$, CTb-labeled neurons in the border region between the nucleus accumbens and the caudate-putamen that are concentrated in patches of strong ENK-IR (arrows in $B$ and $B$ ). $C / C^{\prime}$, Adjacent transverse sections through the rostrolateral part of the nucleus accumbens illustrating the relationship of $\mathrm{CTb}$ labeled neurons $(C)$ with the distribution of ENK-IR $(C)$ following an injection centered in the caudal part of the pars reticulata of the substantia nigra, but involving the pars compacta more rostrally. Arrows mark corresponding blood vessels. Although labeled neurons occur both in- and outside of the large areas of strong ENK-IR surrounding the anterior part of the anterior commissure ( $a c a$ ), the density of neurons is highest inside of the ENK-positive areas (compare with Fig. $6 D, E$ ). $L V$, lateral ventricle. Scale bars, $200 \mu \mathrm{m}$. 


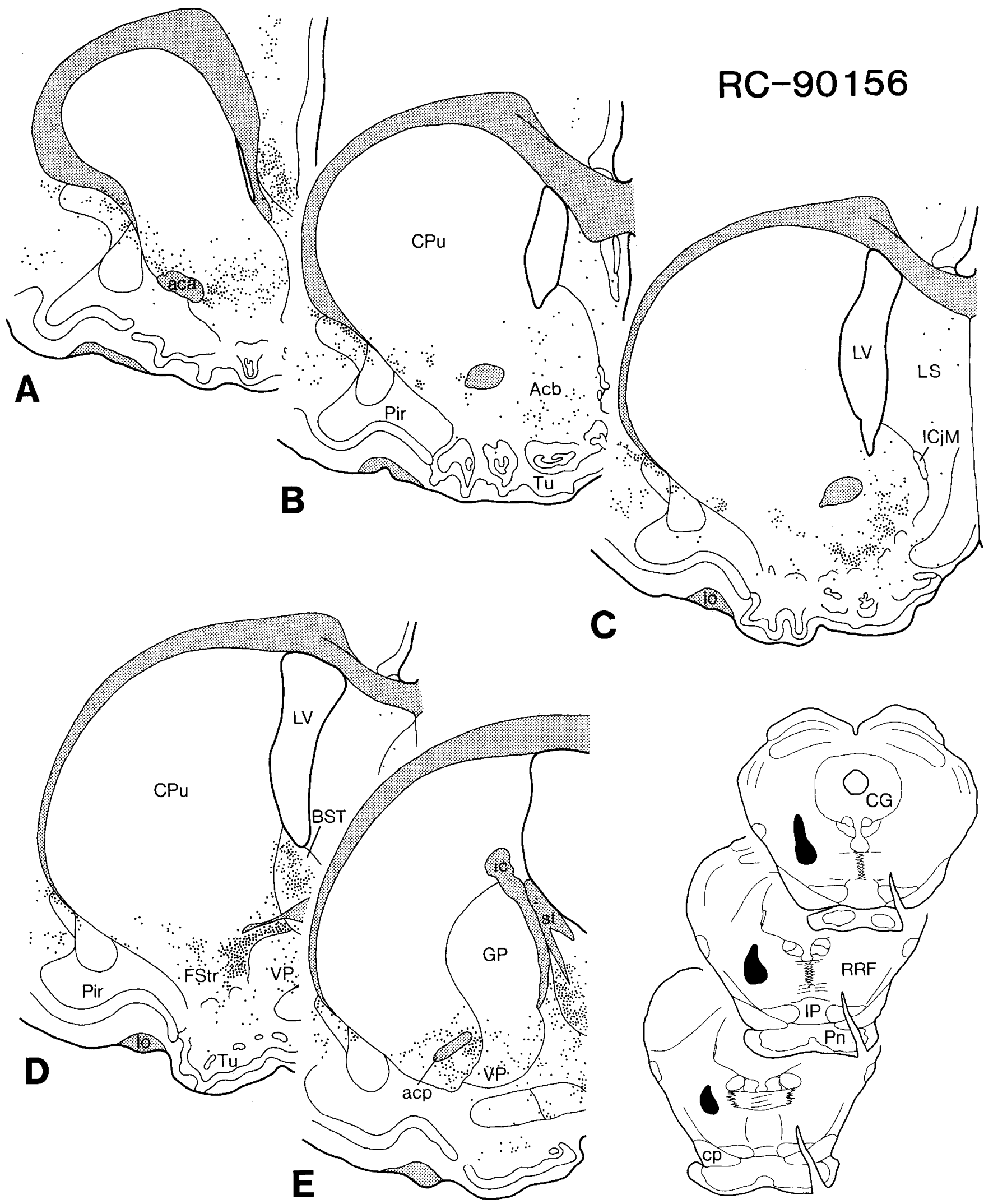

Figure 9. Chartings of retrogradely labeled neurons in five frontal sections through the striatum following an injection of CTb in the retrorubral field $(R R F$; RC-90156). The injection site is drawn in the lower right corner and is shown photographically in Figure $8 A$. Each dot represents a single labcled cell. $C G$, central gray; $I P$, interpeduncular nucleus; $P n$, pontine nuclei; for other abbreviations see Figure 2. 

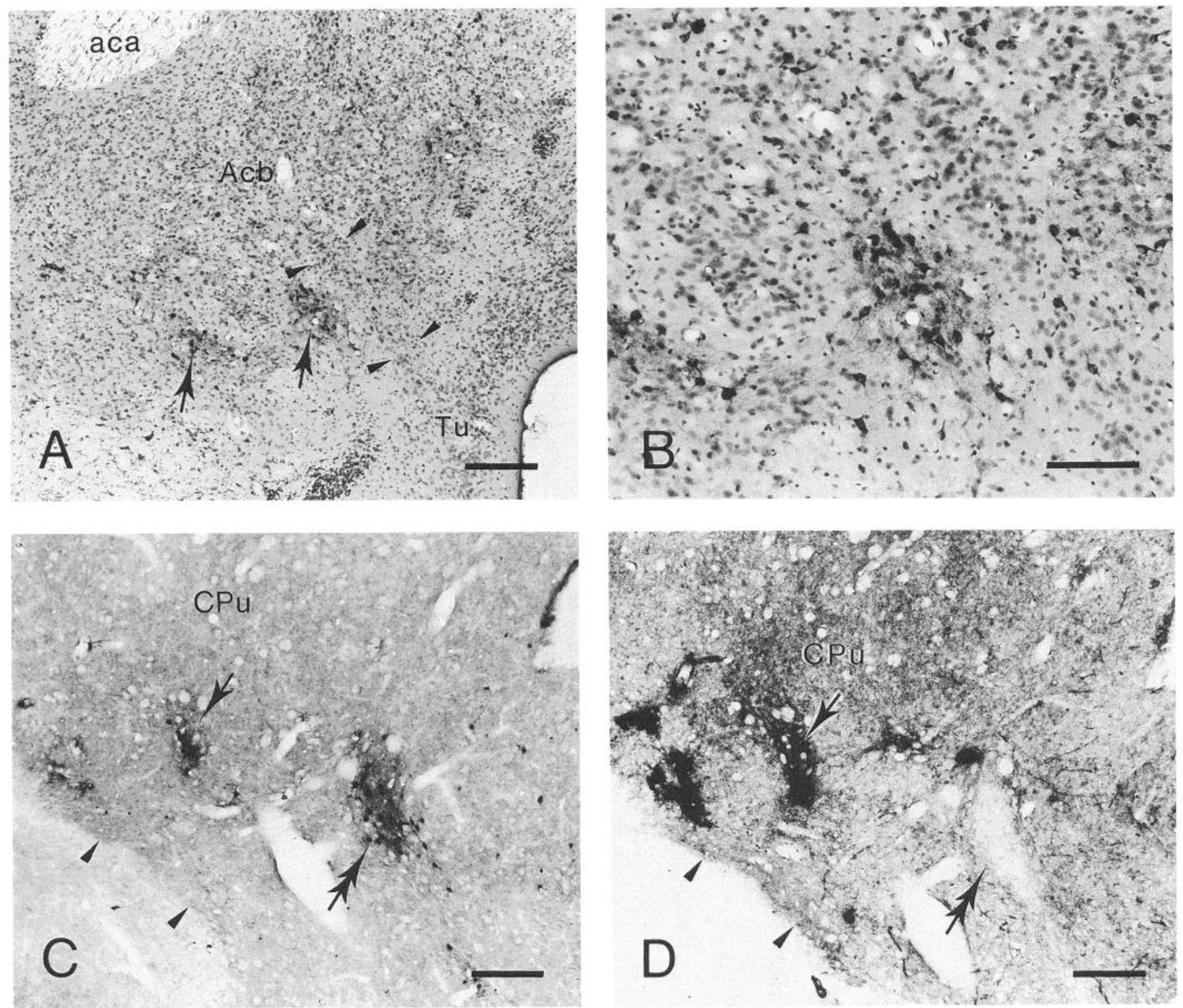

Figure 10. $A$ and $B, \mathrm{CTb}$-labeled neurons in the ventromedial part of the nucleus accumbens $(A c b)$ that result from an injection in the retrorubral field. Both sections are counterstained with cresyl violet. Note in $A$ that groups of labeled neurons at the ventral border of the nucleus accumbens (arrows) are interposed between strands of unlabeled cells that seem to be in continuity with the striatal cell bridges (arrowheads). A higher magnification of the group of labeled neurons in the center of $A$ is illustrated in $B$. At this magnification, it can be appreciated that a narrow zone of low cell density surrounds the group of labeled neurons. $C$ and $D$, Pair of adjacent transverse sections through the ventrolateral part of the caudate-putamen $(C P u)$ to show the relationship between labeled neurons $(C)$ and the distribution of ENK-IR $(D)$ following an injection of $C T$ Tb in the retrorubral field. One group of neurons resides in an area of strong ENK-IR (single arrows in $C$ and $D$ ), whereas another group coincides with an area of very weak or absent ENK-IR (double arrows in $C$ and $D$ ). Arrowheads mark the ventrolateral border of the caudate-putamen. aca, anterior commissure, anterior part; $T u$, olfactory tubercle. Scale bars: $A, C$, and $D, 200 \mu \mathrm{m} ; B, 100 \mu \mathrm{m}$.

dorsomedial part of the shell region of the nucleus accumbens, referred to as the septal pole (Voorn et al., 1986). By contrast, the projections to the lateral part of the ventral tegmental area originate from the ventromedial part of the shell and from the other above-mentioned parts of the ventral striatum. Troiano and Siegel (1978) have provided evidence for a similar topography in the projections from the nucleus accumbens to the ventral tegmental area in the cat.

Substantia nigra pars compacta. The medial part of the substantia nigra receives input from rostral and medial parts of the caudate-putamen and from the core region of the nucleus accumbens, including the rostral parts of this nucleus (cf. Gerfen,
1985). As will be discussed in one of the following paragraphs, distinct populations of striatal neurons in these areas project either to the pars compacta or to the pars reticulata. On the basis of the present data, it cannot be determined whether the efferent targets of the ventromedial part of the shell of the nucleus accumbens include the medial part of the substantia nigra pars compacta. As tracer injections involving only the ventral part of the pars compacta produce virtually no retrograde labeling in the shell, it must be assumed that this projection is restricted to the dorsal parts of the pars compacta. However, all our injections centered in these dorsal parts additionally involve the adjacent ventral tegmental area that receives input 


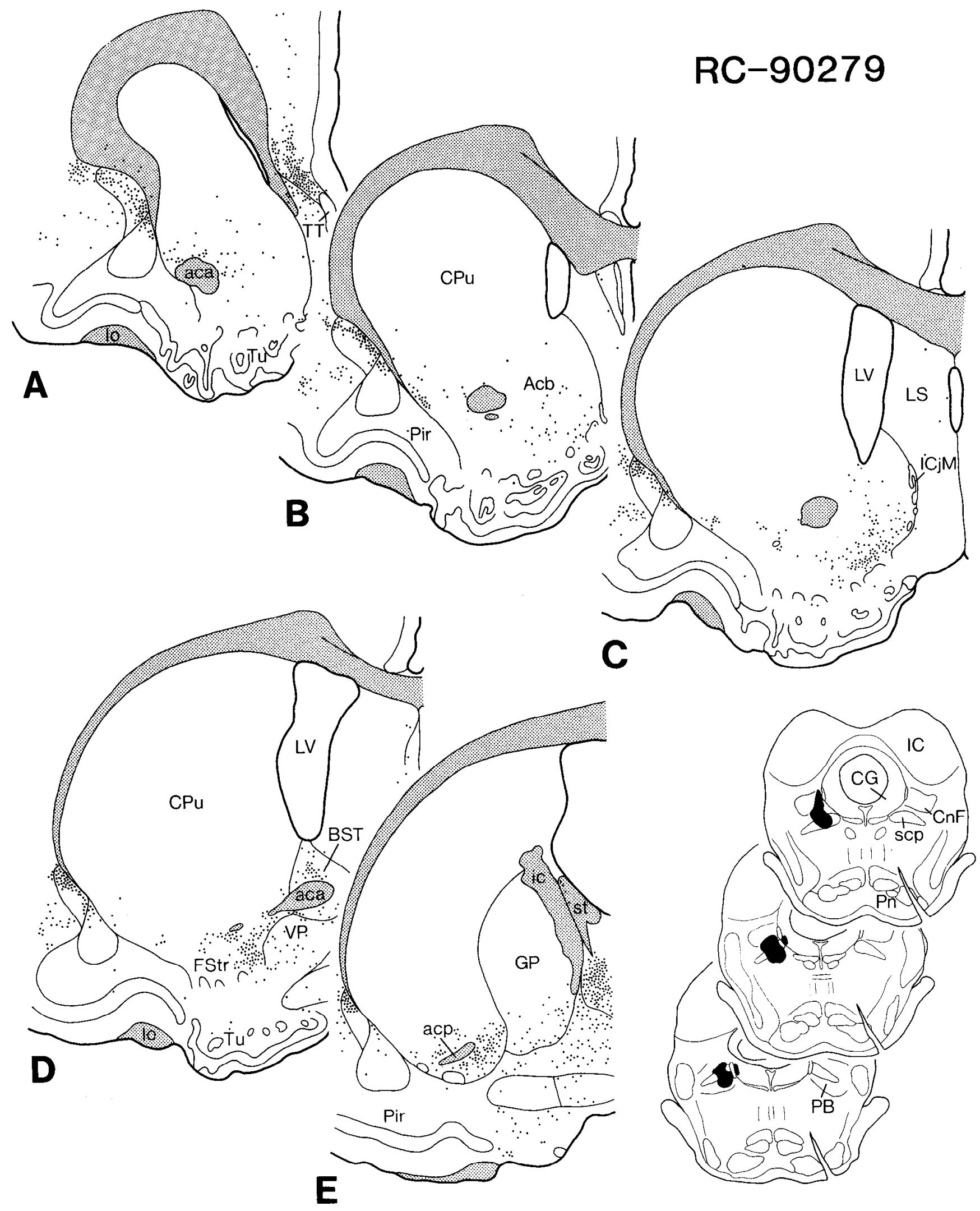

Figure 11. Chartings of retrogradely labeled neurons in five frontal sections through the striatum resulting from an injection of CTb in the peribrachial region (RC-90279). The injection site is drawn in the lower right corner and is shown photographically in Figure 8B. Each dot represents a single labeled cell. $C G$, central gray; $C n F$, cuneiform nucleus; $I C$, inferior colliculus; $P B$, parabrachial nuclei; $P n$, pontine nuclei; $s c p$, superior cerebellar peduncle; for other abbreviations see Figure 2. 

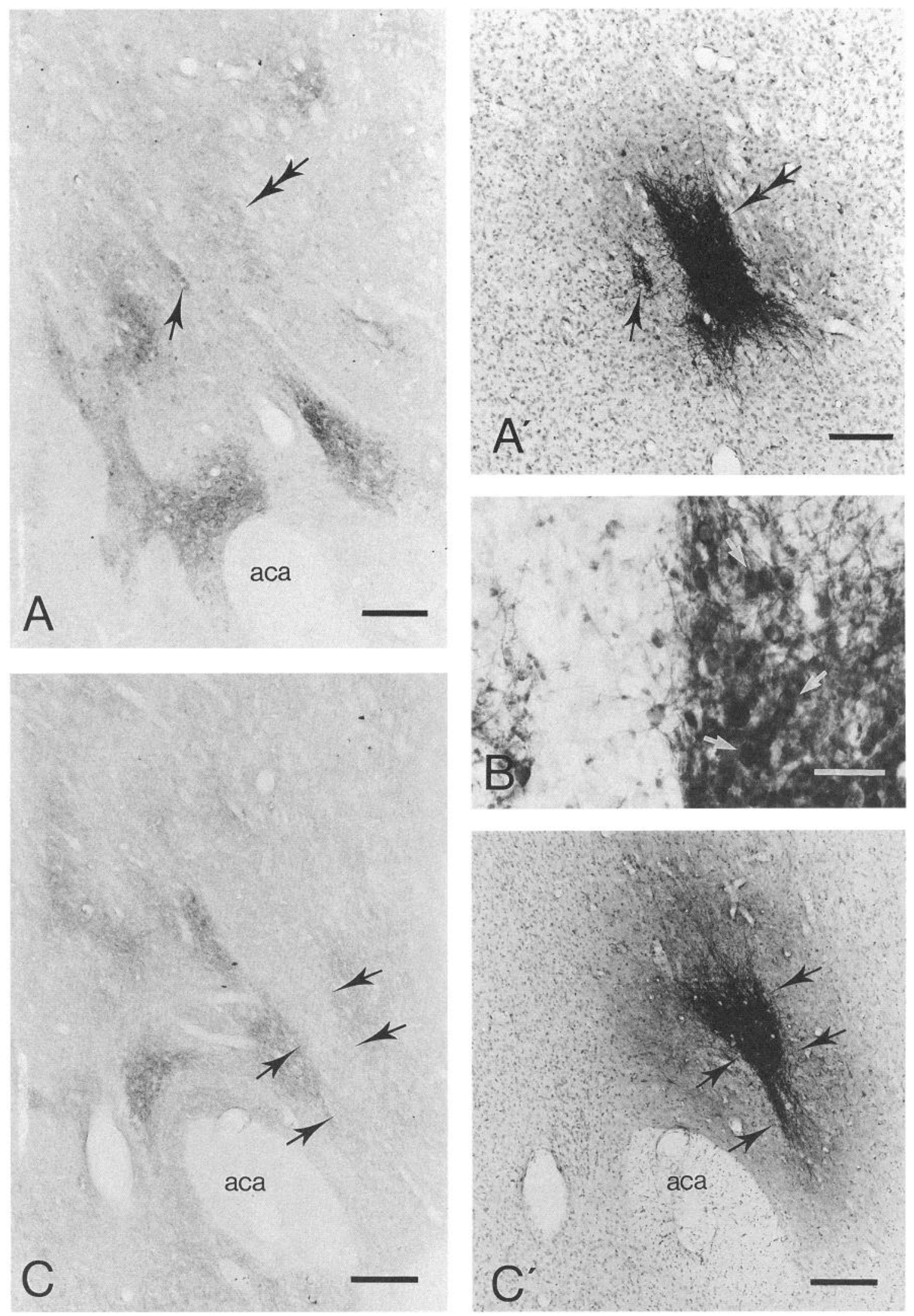

Figure 12. Photomicrographs illustrating the relationships of two PHA-L injection sites $\left(A^{\prime}\right.$ and $C$ ) in the rostrolateral part of the nucleus accumbens with the distribution of ENK-IR in caudally adjacent sections $(A$ and $C)$. Note the peculiar shape of the PHA-L deposits $\left(A^{\prime}\right.$ and $\left.C\right)$. In both cases, a few neurons are labeled quite distant from the injection site. $A / A^{\prime}$, PHA-L injection (RP-90455) that overlaps with an area in which ENK-IR is higher than in the surrounding regions (double arrows). Note that a small group of PHA-L-labeled neurons that lies separate from the remainder of the deposit overlies an equally small area of strong ENK-IR (single arrows in $A$ and $A^{\prime}$; see also Fig. 13A). $B$, Higher magnification of part of the injection site shown in $A^{\prime}$. Note the sharp boundaries of the deposit, which consists of labeled cell bodies (examples indicated by arrows) and dendrites. $C / C^{\prime}$, PHA-L deposit placed in between the large areas of strong ENK-IR that surround the anterior part of the anterior commissure (aca; RP-90480). Arrows in $C$ and $C^{\prime}$ are in corresponding positions (see also Fig. 13B). Scale bars: $A, A^{\prime}, C$, and $C^{\prime}, 200$ $\mu \mathrm{m} ; B, 50 \mu \mathrm{m}$. 


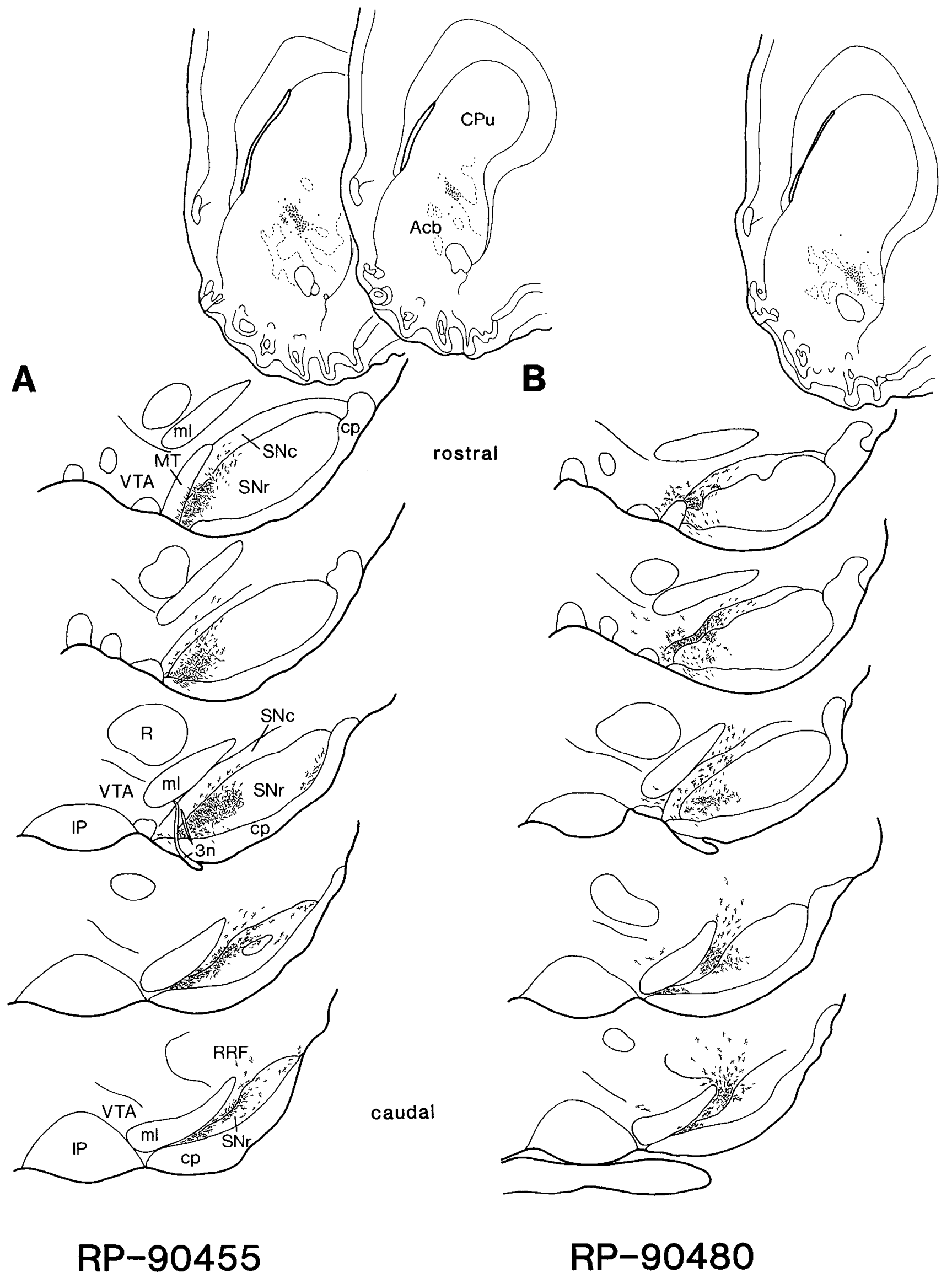


from the ventromedial part of the shell. The existence of a projection from the shell region of the nucleus accumbens to the dorsal parts of the substantia nigra pars compacta would fit in with previous observations that the dorsal tier of the pars compacta shares several characteristics with the ventral tegmental area (Phillipson, 1979a,b).

Retrorubral field. The projections to the retrorubral field, which includes the A8 dopaminergic cell group, originate from neurons in approximately the same striatal areas as the projections to the lateral part of the ventral tegmental area (see above). The majority of these neurons are located in the area surrounding the posterior limb of the anterior commissure and in the fundus striati. A few small groups of neurons in the ventrolateral part of the caudate-putamen are an additional source of input to the retrorubral field. Although projections from the ventral striatum to the retrorubral field or to a comparable region have been reported before in several mammalian species (Conrad and Pfaff, 1976; Nauta et al., 1978; Troiano and Siegel, 1978; Groenewegen and Russchen, 1984; Jiménez-Castellanos and Graybiel, 1989; Haber et al., 1990; Heimer et al., 1991), the present study in the rat is the first to demonstrate their exact origin in the striatum. Unlike the situation in the rat, projections from dorsal parts of the striatum to the retrorubral field have been demonstrated in the cal (Royce and Laine, 1984). The neurons in the ventral striatum that project to the retrorubral area may influence the dopaminergic input to the matrix compartment of the dorsal striatum (Gerfen et al., 1987; cf. Jiménez-Castellanos and Graybiel, 1987; Feigenbaum-Langer and Graybiel, 1989).

Peribrachial region. Anterograde tracing studies have revealed that the projections of the nucleus accumbens extend farther caudally in the mesencephalon than the retrorubral field (Nauta et al., 1978; Troiano and Siegel, 1978; Groenewegen and Russchen, 1984; Haber et al., 1990; Heimer et al., 1991). More in particular, the cuneiform and parabrachial nuclei and the caudoventral parts of the central gray in the rat receive input from this nucleus (Nauta et al., 1978). This peribrachial region may well include the physiologically defined mesencephalic locomotor region (Garcia-Rill, 1986; Garcia-Rill et al., 1987; Coles et al., 1989; see also Rye et al., 1987; Lee et al., 1988). In the present study, the distribution of ventral striatal neurons projecting to this region strikingly resembles that of the neurons issuing fibers to the retrorubral field that contains the A8 cell group. The only difference is that the projections to the peribrachial region from rostral and lateral parts of the ventral striatum seem to be weaker. Involvement of the A8 cell group in the injection site was excluded by comparing sections containing the injection site with adjacent sections stained for tyrosine hydroxylase. Retrograde labeling of neurons in the caudoventral part of the caudate-putamen has been reported in a previous tracing study of the afferents to the peribrachial region (Jackson and Crossman, 1981). In this study, surprisingly few labeled neurons were observed in the nucleus accumbens. The discrepancy with the present results may be attributed either to the superior sensitivity of the CTb tracing technique (Ericson and Blomqvist, 1988) or to the lateral placement of the injections in the study of Jackson and Crossman (1981).

\section{Compartmental distribution of ventral striatal neurons projecting to the mesencephalon}

On the basis of the distribution patterns of a variety of neurochemical substances as well as certain cytoarchitectural features, three regions can be distinguished in the ventral striatum that each display a characteristic compartmental organization (Herkenham et al., 1984; Voorn et al., 1986, 1989; Groenewegen et al., 1989, 1991). Afferents from the prefrontal cortex and the midline and intralaminar nuclei of the thalamus respect the boundaries of the so-defined compartments (Berendse et al., 1988, 1992; Berendse and Groenewegen, 1990). The distribution of the opioid peptide ENK observed in the present material concurs with that reported previously (Berendse et al., 1988; Voorn et al., 1989; Berendse and Groenewegen, 1990) and served as a marker for the ventral striatal compartments. The compartmental relationships of the ventral striatal neurons projecting to the mesencephalon are summarized in Figure 14.

In the first region to be considered, namely, the medial and ventral parts (or shell region) of the nucleus accumbens, cell clusters that coincide with areas of weak ENK-IR are a characteristic feature (Herkenham et al., 1984; Voorn et al., 1989). Neurons in the shell region that project to the ventral tegmental area, the retrorubral field, and the peribrachial region (and possibly to the dorsal tier of the substantia nigra pars compacta) are located outside the cell clusters (Fig. 14), and therefore lie in the compartment receiving afferents from the infralimbic cortex, the paraventricular thalamic nucleus, and the ventral subiculum (Herkenham et al., 1984; Groenewegen et al., 1987; Berendse and Groenewegen, 1990; Berendse et al., 1992). The same afferents may establish contacts with neurons inside the cell clusters, as their dendrites extend up to 50-100 $\mu \mathrm{m}$ outside the clusters (Herkenham et al., 1984). The dendrites of the neurons that project to the mesencephalon seem to avoid entering the cell clusters. Although the CTb method does not produce a complete filling of the dendritic tree, the present results suggest that the inputs from the prelimbic area to the clusters in the medial part of the nucleus accumbens (Donoghue and Herkenham, 1986; Berendse el al., 1992) probably do not establish contacts with neurons projecting to the midbrain. The cells inside of the cell clusters lack projections to the mesencephalon, 


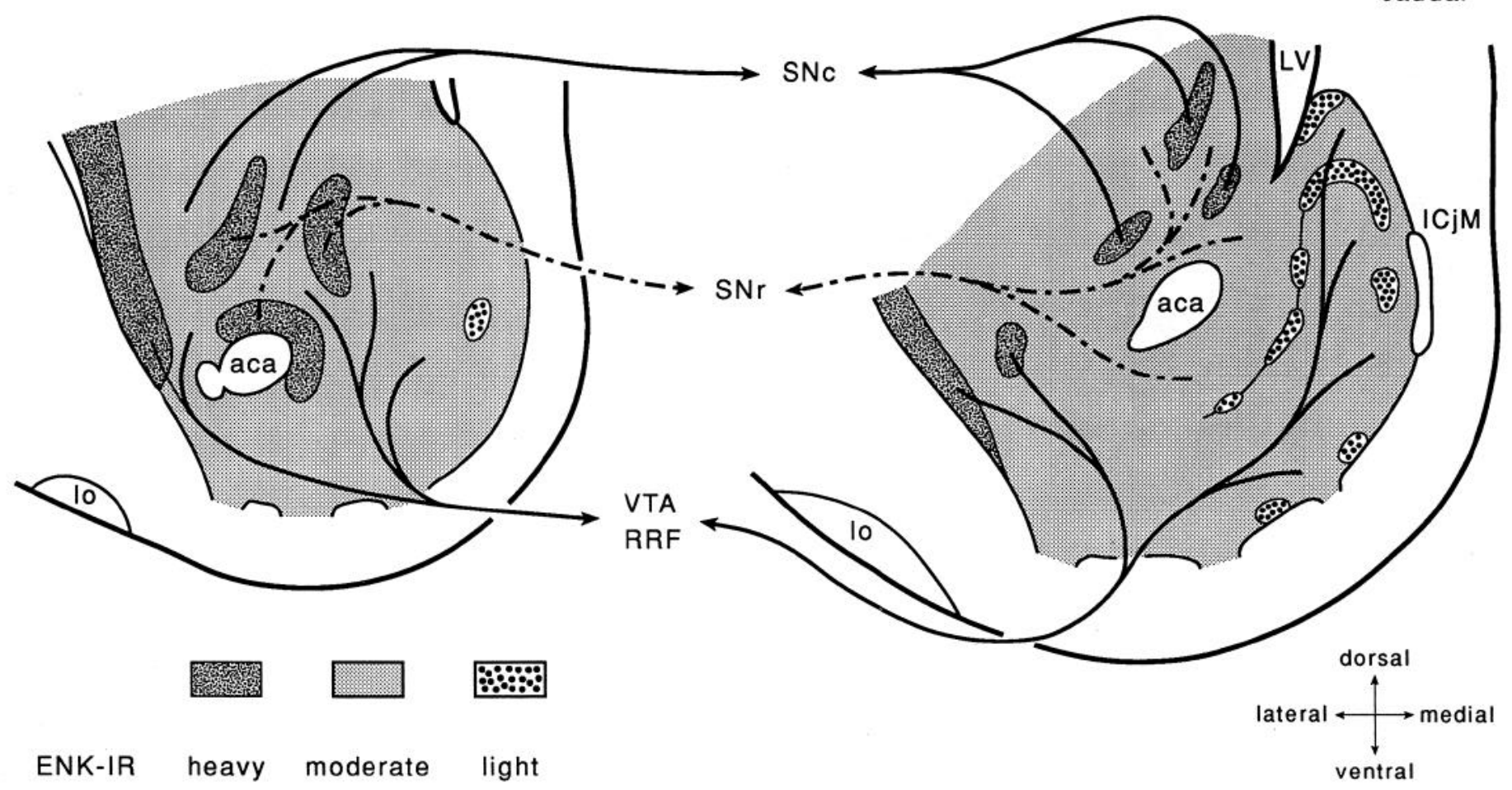

Figure 14. Diagram summarizing the relationships of neurons that project to different targets in the ventral mesencephalon with the compartments in the various sectors of the ventral striatum, identified by ENK immunohistochemistry. Solid lines indicate projections directed toward target areas in which dopaminergic neurons reside, whereas broken lines mark the projections to the substantia nigra pars reticulata (SNr). Minor contributions to a projection are indicated by thin lines. Not indicated in the diagram is the relationship of neurons in the ventromedial part of the shell region with cytoarchitectonic features other than the small cell clusters; for the sake of clarity, the projections to the peribrachial region have not been included in the diagram (for details see Results). aca, anterior commissure, anterior part; $I C j M$, major island of Calleja; $l o$, lateral olfactory tract; $L V$, lateral ventricle; $R R F$, retrorubral field; $S N c$, substantia nigra, pars compacta; $V T A$, ventral tegmental area.

but may, like the cells in the surrounding areas, project to the ventral pallidum $(\mathrm{H}$. W. Berendse and H. J. Groenewegen, unpublished observations). According to Groenewegen et al. (1991), the projections from different parts of the amygdaloid complex either selectively innervate or avoid the cell clusters and may thus influence both output systems. The exact organization of these connections must, however, await electron microscopical investigation.

In the ventral part of the shell region, neurons projecting to mesencephalic targets are not only absent from the cell clusters (Fig. 14), but in addition are heterogeneously distributed over the remainder of the shell region. The neurons appear to avoid strands of cells that are continuous with the striatal cell bridges. At present, these intriguing patterns cannot be related to the organization of the ventral striatal afferents, although fibers from the infralimbic area concentrate near the outer border of the shell as well (Berendse et al., 1992).

In the caudodorsal part of the nucleus accumbens and the adjoining ventral part of the caudate-putamen, patches of strong ENK-IR are embedded in a moderately ENK-IR matrix. By subtracting the results of the retrograde tracer injections not involving the pars reticulata from those that did, it seems possible to conclude that the patches of strong ENK-IR project to the substantia nigra pars compacta, whereas the surrounding matrix sends its fibers to the pars reticulata (Fig. 14). However, as injections in the dorsomedial part of the substantia nigra, which may have involved the pars compacta, resulted in a ho- mogeneous distribution of labeled neurons, an additional projection from the ENK patches to the most dorsal part of the pars reticulata cannot be excluded completely. On the other hand, since the ventral tier dopaminergic neurons extend their dendrites into the pars reticulata (Gerfen et al., 1987), even an injection restricted to the pars reticulata would be expected to label striatal neurons contacting these vertically extending dendrites. As injections of fluorogold in the ventromedial part of the pars reticulata, made in the course of our preliminary experiments (see introductory remarks), produce a matrix pattern of labeling in the striatum similar to that demonstrated by Gerfen $(1984,1985)$, the conclusion drawn above seems justified. The patches of strong ENK-IR receive prefrontal cortical input from deep layer V and layer VI and thalamic input from the paraventricular thalamic nucleus, whereas the matrix is projected upon by layers II through superficial $\mathrm{V}$ of the prefrontal cortex and by the central medial, paracentral, intermediodorsal, and parafascicular thalamic nuclei (Berendse and Groenewegen, 1990; Berendse et al., 1992). Thus, the organization of the cortical inputs and the outputs to the substantia nigra in this part of the ventral striatum is consistent with the arrangement described by Gerfen $(1984,1985,1989$; see also Jiménez-Castellanos and Graybiel, 1989) for the dorsal striatum.

Most of the small groups of neurons in the ventrolateral part of the caudate-putamen that project to the lateral part of the ventral tegmental area, the retrorubral field, and/or the peribrachial region coincide with areas of strong ENK-IR (Fig. 14) 
and may therefore be contacted by fibers from the deep layers of the dorsal agranular insular area and the caudal part of the paraventricular thalamic nucleus (Berendse and Groenewegen, 1990; Berendse et al., 1992). However, in each experiment a single cluster of neurons is located in a conspicuous area from which ENK-IR is virtually absent (Fig. 10C,D). The afferent connections of this area are as yet unknown.

In the most rostrolateral part of the nucleus accumbens (and the adjacent ventrolateral part of the caudate-putamen), several large areas of strong ENK-IR surrounded by moderate ENKIR occur. The large ENK-IR areas coincide with strong calbindin $\mathrm{D}_{28 \mathrm{kDa}}$ immunoreactivity (Voorn et al., 1989). It should be emphasized that these conspicuous compartments are present only in the most rostrolateral part of the ventral striatum; slightly more caudally and medially, ENK-positive areas are smaller and coincide with blanks in the distribution of calbindin $\mathrm{D}_{28 \mathrm{kDa}}$ (Fig. 8 in Voorn et al., 1989). The combined results of our retrograde and anterograde tracing experiments indicate that neurons inside the large ENK-positive areas project to the dorsomedial part of the substantia nigra, whereas projections to the substantia nigra pars compacta and the ventral tegmental area originate from the intervening moderately ENK-IR compartment (Fig. 14). Therefore, the relationships of the two populations of output neurons with the distribution of ENK-IR in the rostrolateral part of the nucleus accumbens are exactly opposite to those found in more caudal parts of the ventral striatum (present results) and in the dorsal striatum (Gerfen, 1984, 1985). As the interrelationships of the distributions of ENK-IR and calbindin $D_{28 \mathrm{kDa}}$ immunoreactivity in the most rostrolateral part of the nucleus accumbens are reversed as well (see above), the present results confirm that calbindin $\mathrm{D}_{28 \mathrm{kDa}}$ immunoreactivity marks compartments that project to the substantia nigra pars reticulata (Gerfen et al., 1985). Neurons projecting to the retrorubral field and the peribrachial region lie for the most part outside of the large areas of strong ENK-IR, but some neurons reside in an area of strong ENK-IR adjacent to the external capsule.

Along the above lines, it seems reasonable to compare the areas of strong ENK-IR in the rostrolateral part of the ventral striatum with the dorsal striatal matrix compartment. Their weak substance P immunoreactivity (Voorn et al., 1989) strengthens this assumption. However, in addition to having some characteristics in common with the matrix compartment, the areas of strong ENK-IR also share a number of characteristics with the patch compartment. Most notably, opiate receptor binding (naloxon) is high in both the patch compartment and in the large ENK-positive areas (A. L. Jongen-Relo, P. Voorn, and H. J. Groenewegen, unpublished observations; see also Herkenham and Pert, 1981; Gerfen et al., 1985, 1987). In addition, both compartments are characterized by weak dopamine immunoreactivity (Voorn et al., 1989) and by thalamic afferents from the paraventricular thalamic nucleus (Berendse et al., 1988; Berendse and Groenewegen, 1990). Lastly, the compartmental relationships of the inputs from the prefrontal cortex in the rostrolateral part of the nucleus accumbens are different from those observed in the caudate-putamen and the caudodorsal part of the nucleus accumbens. In the latter areas, the deep layers of both medial and lateral prefrontal areas project preferentially to the patch compartment, whereas the superficial layers send projections to the matrix compartment (Gerfen, 1989; Berendse et al., 1992). In the rostrolateral part of the nucleus accumbens, fibers from the deep layers of the medial prefrontal cortex likewise reach the compartment projecting to the dopaminergic cell groups, as they terminate in the moderately ENKIR areas (Berendse et al., 1992; present results). However, the fibers from the superficial layers are not as selective and, moreover, terminate preferentially in the same compartment as those from the deeper layers (Berendse et al., 1992). The projections from the lateral prefrontal areas to the rostrolateral part of the nucleus accumbens show a similar lack of compartmental selectivity. Thus, assigning a patch or matrix identity to the compartments in the rostrolateral part of the nucleus accumbens can only be achieved by using a restricted set of criteria. If we instead consider all of the connections and neurochemical features discussed above, these compartments share characteristics with both patch and matrix compartments.

The analomical data discussed above indicate that the compartments in the shell region and in the most rostrolateral part of the nucleus accumbens have unique combinations of immunohistochemical characteristics and input/output relations, different from those of the patch/matrix compartments in other parts of the striatum. Moreover, in sharp contrast with the strictly reciprocal connections of the dorsal striatal patches with a specific subgroup of nigral dopaminergic neurons (Gerfen et al., 1987), the projections of the above-mentioned ventral striatal compartments to the $A 8, \Lambda 9$, and $\Lambda 10$ dopaminergic cell groups have primarily an open loop, feedforward character (Nauta et al., 1978; present results).

In summarizing the input/output relations described above for the three main ventral striatal regions, a remarkable conclusion can be drawn. When the ventral striatomesencephalic projection is considered as a dual system, that is, consisting of a component projecting to the substantia nigra pars compacta and associated dopaminergic cell groups and a component projecting to the nondopaminergic elements of the nigral complex, the thalamic paraventricular nucleus may affect both components. Thus, through its strong innervation of the shell region of the nucleus accumbens and via its projections to the small ENK-rich patches in the border region between the ventral part of the caudate-putamen and the dorsal part of the nucleus accumbens, the paraventricular nucleus may influence all dopaminergic cell groups in the nigral complex. By way of the large ENK-positive areas in the rostrolateral part of the nucleus accumbens, the paraventricular nucleus may further reach a restricted part of the pars reticulata of the substantia nigra. In the rostrolateral part of the nucleus accumbens and more caudally in the border region between the nucleus accumbens and the caudate-putamen, the projections from other nuclei of the midline and intralaminar thalamic nuclear complex, most notably the central medial, paracentral, intermediodorsal, and parafascicular nuclei, complement those of the paraventricular nucleus (Berendse and Groenewegen, 1990). Thus, in these areas the paraventricular influence on one of the two components of the striatomesencephalic system may be counterbalanced by an influence from presumably functionally different thalamic nuclei on the other component of the system. In this respect, the thalamic inputs to the ventral striatum differ from those of the prefrontal cortex, discussed in a preceding paragraph. The functional significance of these differential arrangements of thalamic and prefrontal cortical projections with respect to the output of the ventral striatum remains to be established. A highly speculative interpretation is that a single input, for example, from the paraventricular thalamic nucleus, may have diverse or even opposing effects on the output of the basal 
ganglia through different striatal regions. On the other hand, the effects of a particular input may be strongly influenced by the other afferents to the same compartment in the striatal region under consideration, as well as by the presence of a specific combination of neuromodulating factors of extrinsic or intrinsic origin, reflected in the neurochemical characteristics of the compartment.

\section{Regional and subregional heterogeneity of ventral striatal efferents}

The compartmental or subregional origin of the projections from the ventral striatum determines whether the fibers reach dopaminergic or nondopaminergic targets in the midbrain. Some degree of topography is present in the projections to the dopaminergic cell groups (present results), but topographical principles may be more obvious in the projections to the pars reticulata of the substantia nigra. However, Haber et al. (1990) have reported that in the monkey the topographical ordering in the projections from the ventral striatum to the substantia nigra pars reticulata is not as strict as that in the ventral striatopallidal projections. Rather strict mediolateral and dorsoventral coordinates are maintained in the latter projections (Mogenson et al., 1983; Haber et al., 1990; Zahm and Heimer, 1990; Heimer et al., 1991). It is as yet unclear whether compartmental differences, similar to those reported for the dorsal striatum (JiménezCastellanos and Graybiel, 1989; Giménez-Amaya and Graybiel, 1990), exist in the ventral striatopallidal projections as well.

Taking all of these data into consideration, the ventral striatopallidal pathway is primarily involved in parallel output pathways relaying specific sets of cortical and thalamic inputs via the pallidum and the mediodorsal thalamus ultimately back to the cortex (Groenewegen and Berendse, 1990; Groenewegen et al., 1990b; cf. Alexander et al., 1986, 1990). By contrast, the component of the ventral striatomesencephalic system reaching the dopaminergic cell groups A10, A9, and A8 may play an important role in modulating the dopaminergic input to virtually the entire striatum (Fallon and Moore, 1978; Björklund and Lindvall, 1984; Gerfen et al., 1987). The other component can, by way of the medial part of the pars reticulata of the substantia nigra, participate in nigrothalamic and nigrotectal output pathways of the basal ganglia (Beckstead et al., 1981; Beckstead, 1983; Williams and Faull, 1985).

\section{Limbic influences on motor behavior}

The association of the nucleus accumbens with the limbic system and its close relationship with the caudate-putamen, traditionally considered to play a prominent role in motor functions, has led to the notion that this nucleus mediates limbic influences on motor behavior (Mogenson and Yim, 1981). The projection from the nucleus accumbens to the dopaminergic neurons that innervate the caudate-putamen has been suggested as a possible anatomical substrate for this influence (Nauta et al., 1978; Somogyi et al., 1981; Nauta and Domesick, 1984). Furthermore, the nucleus accumbens may influence motor behavior by way of its projections to the mesencephalic locomotor region via the ventral pallidum (Mogenson and Nielsen, 1983, 1984; Mogenson et al., 1989). A third pathway via which the nucleus accumbens may affect motor behavior leads through the ventral pallidum and the mediodorsal nucleus to the prefrontal cortex, which in turn is connected with the premotor cortex (Heimer et al., 1982). However, since the projections from limbic brain areas such as the hippocampal formation and the amygdala include large parts of the caudate-putamen (Kelley and Domesick, 1982; Kelley et al., 1982; Russchen and Price, 1984; Russchen et al., 1985; Groenewegen et al., 1987; Kita and Kitai, 1990), it is clear that the nucleus accumbens can no longer be regarded as the only limbic-related part of the striatum. Furthermore, the results of recent anatomical, physiological, and behavioral investigations indicate that the dichotomy "limbicrelated ventral striatum" versus "motor-related dorsal striatum" does not adequately describe the high degree of functional specialization within the striatum (Oberg and Divac, 1979; Dunnett and Iversen, 1980, 1981, 1982a-c; Alexander et al., 1986, 1990; Rolls and Williams, 1987; Kelley et al., 1989; Groenewegen et al., 1990b; Pisa and Cyr, 1990). Several sectors can be identified in the striatum that are involved in separate parallel cortico-striato-pallido-thalamo-cortical circuits and may subserve functions ranging from simple motor acts to the most complex behaviors (Alexander et al., 1986, 1990; Groenewegen et al., 1990b). Furthermore, the present results reveal that the influence of the nucleus accumbens on the dopaminergic innervation of most of the striatum, as suggested already by Nauta et al. (1978), is exerted by a specific subpopulation of neurons in the ventral striatum that receive a characteristic set of afferents.

In view of the above-described data, the notion that the nucleus accumbens represents a limbic-motor interface, true as this might be, is an underestimate of the potential influences of this structure on behavior. Therefore, it would seem more accurate to focus on the very specific population of neurons that can influence the dopaminergic innervation of virtually the entire striatum. Most of these neurons reside in the shell region of the nucleus accumbens and in the fundus striati, regions that receive prominent inputs from the paraventricular thalamic nucleus, the ventral subiculum, and the infralimbic and ventral prelimbic cortical areas (Herkenham et al., 1984; Groenewegen et al., 1987; Berendse and Groenewegen, 1990; Berendse et al., 1992), which are involved in the mediation of autonomic responses (Terreberry and Neafsey, 1984; Hurley-Gius and Neafsey, 1986; I Iardy and Holmes, 1988; Buchanan et al., 1989). Thus, the projections of this subpopulation of ventral striatal neurons may provide a pathway for visceral or autonomic influences on behavior.

\section{References}

Alexander GE, DeLong MR, Strick PL (1986) Parallel organization of functionally segregated circuits linking basal ganglia and cortex. Annu Rev Neurosci 9:357-381.

Alexander GE, Crutcher MD, DeLong MR (1990) Basal ganglia-thalamocortical circuits: parallel substrates for motor, oculomotor, "prefrontal' and 'limbic' functions. In: Progress in Brain Research, Vol 85, The prefrontal cortex: its structure, function, and pathology (Uylings HBM, Van Eden CG, De Bruin JPC, Corner MA, Feenstra MGP, eds), pp 119-146. Amsterdam: Elsevier.

Arts MPM, Groenewegen H.I (1992) Relationship of the dendritic arborizations of ventral striato-mesencephalic projection neurons with boundaries of striatal compartments. An in vitro intracellular labeling study in the rat. Eur $\mathbf{J}$ Neurosci, in press.

Beckstead RM (1983) Long collateral branches of substantia nigra pars reticulata axons to thalamus, superior colliculus and reticular formation in monkey and cat. Multiple retrograde neuronal labeling with fluorescent dyes. Neuroscience 10:767-779.

Beckstead RM, Edwards SB, Frankfurter A (1981) A comparison of the intranigral distribution of nigrotectal neurons labeled with horseradish peroxidase in the monkey, cat and rat. J Neurosci 1:121-125.

Berendse HW, Groenewegen HJ (1990) Organization of the thalamostriatal projections in the rat, with special emphasis on the ventral striatum. J Comp Neurol 299:187-228. 
Berendse HW, Groenewegen HJ (1991) Restricted cortical termination fields of the midline and intralaminar thalamic nuclei in the rat. Neuroscience 42:73-102.

Berendse HW, Voorn P, te Kortschot A, Groenewegen HJ (1988) Nuclear origin of thalamic afferents of the ventral striatum determines their relation to patch/matrix configurations in enkephalin-immunoreactivity in the rat. J Chem Neuroanat 1:3-10.

Berendse HW, Galis-de Graaf Y, Groenewegen HJ (1992) Topographical organization and relationship with ventral striatal compartments of prefrontal corticostriatal projections in the rat. J Comp Neurol 316: 314-347.

Björklund A, Lindvall O (1984) Dopamine-containing systems in the CNS. In: Handbook of chemical neuroanatomy, Vol 2, Classical neurotransmitters in the CNS (Björklund A, Hökfelt T, eds), pp 55-122. Amstcrdam: Elsevicr.

Brutus M, Zuabi S, Siegel A (1989) Microinjections of D-ALA²-Met' ${ }^{5}$ enkephalinamide placed into nucleus accumbens suppress hypothalamically elicited hissing in the cat. Exp Neurol 104:55-61.

Buchanan SL, Thompson RH, Powell DA (1989) Midline thalamic lesions enhance conditioned bradycardia and the cardiac orienting reflex in rabbits. Psychobiology 17:300-306.

Coles SK, Iles JF, Nicolopoulos-Stournaras S (1989) The mesencephalic centre controlling locomotion in the rat. Neuroscience 28:149157.

Conrad LCA, Pfaff DW (1976) Autoradiographic tracing of nucleus accumbens efferents in the rat. Brain Res 113:589-596.

Dahlström A, Fuxe K (1964) Evidence for the existence of monoamine-containing neurons in the central nervous system. I. Demonstration of monoamines in the ccll bodics of brain stem neurons. Acta Physiol Scand 62: [Suppl 232] 1-55.

Donoghue JP, Herkenham M (1986) Neostriatal projections from individual cortical fields conform to histochemically distinct striatal compartments in the rat. Brain Res 365:397-403.

Dunnett SB, Iversen SD (1980) Regulatory impairments following selective kainic acid lesions of the neostriatum. Behav Brain Res 1: 497-506.

Dunnett SB, Iversen SD (1981) Learning impairments following selective kainic acid-induced lesions within the neostriatum of rats. Behav Brain Res 2:189-209.

Dunnett SB, Iversen SD (1982a) Regulatory impairments following selective 6-OHDA lesions of the neostriatum. Behav Brain Res 4: 195-202.

Dunnett SB, Iversen SD (1982b) Neurotoxic lcsions of ventrolatcral but not anteromedial neostriatum impair differential reinforcement of low rates (DRL) performance. Behav Brain Res 6:213-226.

Dunnett SB, Iversen SD (1982c) Sensorimotor impairments following localized kainic acid and 6-hydroxydopamine lesions of the neostriatum. Brain Res 248:121-127.

Ericson H, Blomqvist A (1988) Tracing of neuronal connections with cholera toxin subunit B: light and electron microscopic immunohistochemistry using monoclonal antibodies. J Neurosci Methods 24:225235.

Evans KR, Vaccarino FJ (1986) Intra-nucleus accumbens amphetamine: dose-dependent effects on food intake. Pharmacol Biochem Behav 25:1149-1151.

Fallon JH, Moore RY (1978) Catecholamine innervation of the basal forebrain. IV. Topography of the dopamine projection to the basal forebrain and neostriatum. J Comp Neurol 180:545-580.

Feigenbaum-Langer L, Graybiel AM (1989) Distinct nigrostriatal projection systems innervate striosomes and matrix in the primate striatum. Brain Res 498:344-350.

Garcia-Rill E (1986) The basal ganglia and the locomotor regions. Brain Res Rev 11:47-63.

Garcia-Rill E, Houser CR, Skinner RD, Smith W, Woodward DJ (1987) Locomotion-inducing sites in the vicinity of the pedunculopontine nucleus. Brain Res Bull 18:731-738.

Gerfen CR (1984) The neostriatal mosaic: compartmentalization of corticostriatal input and striatonigral output systems. Nature 311: $461-464$

Gerfen CR (1985) The neostriatal mosaic. I. Compartmental organization of projections from the striatum to the substantia nigra in the rat. J Comp Neurol 236:454-476.

Gerfen CR (1989) The neostriatal mosaic: striatal patch-matrix organization is related to cortical lamination. Science 246:385-388.

Gerfen CR, Sawchencko PE (1984) An anterograde neuroanatomical tracing method that shows the detailed morphology of neurons, their axons and terminals: immunohistochemical localization of an axonally transported plant lectin, Phaseolus vulgaris-leucoagglutinin (PHA-L). Brain Res 290:219-238.

Gerfen CR, Baimbridge KG, Miller JJ (1985) The neostriatal mosaic compartmental distribution of calcium-binding protein and parvalbumin in the basal ganglia of the rat and monkey. Proc Natl Acad Sci USA 82:8780-8784.

Gerfen CR, Herkenham M, Thibault J (1987) The neostriatal mosaic. II. Patch- and matrix-directed mesostriatal dopaminergic and nondopaminergic systems. J Neurosci 7:3915-3934.

Giménez-Amaya JM, Graybiel AM (1990) Compartmental origins of the striatopallidal projection in the primate. Neuroscience $34: 111-$ 126

Graybiel $\Lambda \mathrm{M}$ (1984) Neurochemically specified subsystems in the basal ganglia. In: Ciba Foundation symposium 107, Functions of the basal ganglia (Evered D, O'Connor M, eds), pp 114-149. London: Pitman.

Graybiel AM (1990) Neurotransmitters and neuromodulators in the basal ganglia. Trends Neurosci 13:244-254.

Graybiel AM, Ragsdale CW Jr (1978) Histochemically distinct compartments in the striatum of human, monkey, and cat demonstrated by acetylcholinesterase staining. Proc Natl Acad Sci USA 75:57235726.

Graybiel AM, Ragsdale CW Jr, Yoneoka ES, Elde RP (1981) An immunohistochemical study of enkephalin and other neuropeptides in the striatum of the cat with evidence that the opiate peptides are arranged to form mosaic patterns in register with the striosomal compartments visible by acetylcholinesterase staining. Neuroscience 6: 377-397.

Groenewegen HJ, Berendse HW (1990) Parallel arrangement of forebrain circuits in the rat. 2. Ventral striatum, ventral pallidum, and mediodorsal thalamic nucleus. Soc Neurosci Abstr 16:426.

Groenewegen HJ, Russchen FT (1984) Organization of the efferent projections of the nucleus accumbens to pallidal, hypothalamic, and mesencephalic structures: a tracing and immunohistochemical study in the cat. J Comp Neurol 223:347-367.

Groenewegen HJ, Vermeulen-Van der Zee E, te Kortschot A, Witter MP (1987) Organization of the projections from the subiculum to the ventral striatum in the rat. A study using anterograde transport of Phaseolus vulgaris-leucoagglutinin. Neuroscience 23:103-120.

Groenewegen HJ, Meredith GE, Berendse HW, Voorn P, Wolters JG (1989) The compartmental organization of the ventral striatum in the rat. In: Current problems in neurology, Vol 9, Neural mechanisms in disorders of movement (Crossman AR, Sambrook MA, eds), pp 45-54. London: Libbey.

Groenewegen HJ, Arts MPM, Berendse HW (1990a) A cellular basis for the compartmental structure of the ventral striatum in the rat. Eur J Neurosci [Suppl] 3:294.

Groenewegen HJ, Berendse HW, Wolters JG, Lohman AHM (1990b) The anatomical relationship of the prefrontal cortex with the striatopallidal system, the thalamus and the amygdala: evidence for a parallel organization. In: Progress in Brain Research, Vol 85, The prefrontal cortex: its structure, function, and pathology (Uylings HBM, Van Eden CG, De Bruin JPC, Corner MA, Feenstra MGP, eds), pp 95-118. Amsterdam: Elsevier.

Groenewegen HJ, Berendse HW, Meredith GE, Haber SN, Voorn P, Wolters JG, Lohman AHM (1991) Functional anatomy of the ventral, limbic system-innervated striatum. In: The mesolimbic dopamine system: from motivation to action (Willner $\mathbf{P}$, Scheel-Krüger $J$, eds), pp 19-59. Chichester: Wiley.

Haber SN, Lynd E, Klein C, Groenewegen HJ (1990) Topographic organization of the ventral striatal efferent projections in the rhesus monkey: an anterograde tracing study. J Comp Neurol 293:282-298.

Hardy SGP. Holmes DE (1988) Prefrontal stimulus-produced hypotension in rat. Exp Brain Res 73:249-255.

Heimer L, Wilson RD (1975) The subcortical projections of the allocortex: similarities in the neural associations of the hippocampus, the piriform cortex, and the neocortex. In: Golgi centennial symposium: perspectives in neurobiology (Santini M, ed), pp 177-193. New York: Raven.

Heimer L, Switzer RD, VanHoesen GW (1982) Ventral striatum and ventral pallidum. Components of the motor system? Trends Neurosci 5:83-87.

Heimer L, Zahm DS, Churchill L, Kalivas PW, Wohltman C (1991) 
Specificity in the projection patterns of accumbal core and shell in the rat. Neuroscience 41:89-125.

Herkenham M, Pert CB (1981) Mosaic distribution of opiate receptors, parafascicular projections and acetylcholinesterase in rat striatum. Nature 291:415-418.

Herkenham M, Moon Edley S, Stuart J (1984) Cell clusters in the nucleus accumbens of the rat, and the mosaic relationship of opiate receptors, acetylcholinesterase and subcortical afferent terminations. Neuroscience 11:561-593.

Hurley-Gius KM, Neafsey EJ (1986) The medial frontal cortex and gastric motility: microstimulation results and their possible significance for the overall pattern of organization of rat frontal and parietal cortex. Brain Res 365:241-248.

Jackson A, Crossman AR (1981) Basal ganglia and other afferent projections to the peribrachial region in the rat: a study using retrograde and anterograde transport of horseradish peroxidase. Neuroscience 6:1537-1549.

Jiménez-Castellanos J, Graybiel AM (1987) Subdivisions of the dopamine-containing A8-A9-A10 complex identified by their differential mesostriatal innervation of striosomes and extrastriosomal matrix. Neuroscience 23:223-242.

Jiménez-Castellanos J, Graybiel AM (1989) Compartmental origins of striatal efferent projections in the cat. Neuroscience 32:297-321.

Kawaguchi Y, Wilson CJ, Emson PC (1989) Intracellular recording of identified neostriatal patch and matrix spiny cells in a slice preparation preserving cortical inputs. J Neurophysiol 62:1052-1068.

Kelley AE, Domesick VB (1982) The distribution of the projection from the hippocampal formation to the nucleus accumbens in the rat: an anterograde- and retrograde-horseradish peroxidase study. Neuroscience $7: 2321-2335$.

Kelley AE, Domesick VB, Nauta WJH (1982) The amygdalostriatal projection in the rat-an anatomical study by anterograde and retrograde tracing methods. Neuroscience 7:615-630.

Kelley AE, Gauthier AM, Lang CG (1989) Amphetamine microinjections into distinct striatal subregions cause dissociable effects on motor and ingestive behavior. Behav Brain Res 35:27-39.

Kita H, Kitai ST (1990) Amygdalostriatal projections to the frontal cortex and the striatum in the rat. J Comp Neurol 298:40-49.

Lee HJ, Rye DB, Hallanger AE, Levey AI, Wainer BH (1988) Cholinergic vs. noncholinergic efferents from the mesopontine tegmentum to the extrapyramidal motor system nuclei. J Comp Neurol 275:469492.

Lorens SA, Sorensen JP, Harvey JA (1970) Lesions in the nuclei accumbens septi of the rat. J Comp Physiol Psychol 73:284-290.

Martin LJ, Hadfield MG, Dellovade TL, Price DL (1991) The striatal mosaic in primates: patterns of neuropeptide immunoreactivity differentiate the ventral striatum from the dorsal striatum. Neuroscience 43:397-417.

Mogenson GJ, Nielsen MA (1983) Evidence that an accumbens to subpallidal GABAergic projection contributes to locomotor activity. Brain Res Bull 11:309-314.

Mogenson GJ, Nielsen MA (1984) Neuropharmacological evidence to suggest that the nucleus accumbens and subpallidal region contribute to exploratory locomotion. Behav Neural Biol 42:52-60.

Mogenson GJ, Wu M (1988) Disruption of food hoarding by injections of procaine into mediodorsal thalamus, GABA into subpallidal region and haloperidol into accumbens. Brain Res Bull 20:247-251.

Mogenson GJ, Yim CY (1981) Electrophysiological and neuropharmacological-behavioral studies of the nucleus accumbens: implications for its role as a limbic-motor interface. In: The neurobiology of the nucleus accumbens (Chronister RB, DeFrance JF, eds), pp 210 229. Brunswick: Haer Institute.

Mogenson GJ, Swanson I,W, Wu M (1983) Neural projections from nucleus accumbens to globus pallidus, substantia innominata, and lateral preoptic-lateral hypothalamic area: an anatomical and electrophysiological investigation in the rat. J Neurosci 3:189-202.

Mogenson GJ, Wu M, Tsai CT (1989) Subpallidal-pedunculopontine projections but not subpallidal-mediodorsal thalamus projections contribute to spontaneous locomolor activity. Brain Res 485:396398.

Nauta WJH (1986) Circuitous connections linking cerebral cortex, limbic system, and corpus striatum. In: The limbic system: functional organization and clinical disorders (Doane BK, Livingston KE, eds), pp 67-77. New York: Raven.

Nauta WJH, Domesick VB (1984) Afferent and efferent relationships of the basal ganglia. In: Ciba Foundation symposium 107, Functions of the basal ganglia (Evered D, O'Connor M, eds), pp 3-29. London: Pitman.

Nauta WJH, Smith GP, Faull RLM, Domesick VB (1978) Efferent connections and nigral afferents of the nucleus accumbens septi in the rat. Neuroscience $3: 385-401$.

Oberg RGE, Divac I (1979) "Cognitive" functions of the neostriatum. In: The neostriatum (Divac I, Oberg RGE, eds), pp 291-313. Oxford: Pergamon.

Paxinos G, Watson C (1986) The rat brain in stereotaxic coordinates, 2d ed. Sydney: Academic.

Penny GR, Wilson CJ, Kitai ST (1988) Relationship of the axonal and dendritic geometry of spiny projection neurons to the compartmental organization of the neostriatum. J Comp Neurol 269:275289.

Phillips AG, Brooke SM, Fibiger HC (1975) Effects of amphetamine isomers and neuroleptics on self-stimulation from the nucleus accumbens and dorsal noradrenergic bundle. Brain Res 85:13-22.

Phillipson OT (1979a) The cytoarchitecture of the interfascicular nucleus and ventral tegmental area of Tsai in the rat. J Comp Neurol $187: 85-98$.

Phillipson OT (1979b) A Goldi study of the ventral tegmental area of Tsai and interfascicular nucleus in the rat. J Comp Neurol 187:99116.

Phillipson OT (1979c) Afferent projections to the ventral tegmental area of Tsai and interfascicular nucleus: a horseradish peroxidase study in the rat. J Comp Neurol 187:117-144.

Pijnenburg AJJ, Van Rossum JM (1973) Stimulation of locomotor activity following injection of dopamine into the nucleus accumbens. J Pharm Pharmacol 25:1003-1005.

Pisa M, Cyr J (1990) Regionally selective roles of the rat's striatum in modality-specific discrimination learning and forelimb reaching. Behav Brain Res 37:281-292.

Ragsdale CW Jr, Graybiel AM (1981) The fronto-striatal projection in the cat and monkey and its relationship to inhomogeneities established by acetyl-cholinesterase histochemistry. Brain Res 208:259 266.

Ragsdale CW Jr, Graybiel AM (1990) A simple ordering of neocortical areas established by the compartmental organization of their striatal projections. Proc Natl Acad Sci USA 87:6196-6199.

Rolls ET, Williams GV (1987) Sensory and movement-related neuronal activity in different regions of the primate striatum. In: Basal ganglia and behavior: sensory aspects of motor functioning (Schneider JS, Lidsky TI, eds), pp 37-59. Toronto: Huber.

Royce GJ, Laine EJ (1984) Efferent connections of the caudate nucleus, including cortical projections of the striatum and other basal ganglia: an autoradiographic and horseradish peroxidase investigation in the cat. J Comp Neurol 226:28-49.

Russchen FT, Price IL (1984) Amygdalostriatal projections in the rat. Topographical organization and fiber morphology shown using the lectin PHA-L as an anterograde tracer. Neurosci Lett 47:15-22.

Russchen FT, Bakst I, Amaral DG, Price JL (1985) The amygdalostriatal projections in the monkey: an anterograde tracing study. Brain Res 329:241-258.

Rye DB, Saper CB, Lee HJ, Wainer BH (1987) Pedunculopontine tegmental nucleus of the rat: cytoarchitecture, cytochemistry, and some extrapyramidal connections of the mesopontine tegmentum. $J$ Comp Neurol 259:483-528.

Smith MO, Holland RC (1975) Effects of lesions of the nucleus accumbens on lactation and postpartum behavior. Physiol Psychol 3: 331-336.

Somogyi P, Bolam JP, Totterdell S, Smith AD (1981) Monosynaptic input from the nucleus accumbens-ventral striatum region to retrogradely labelled nigrostriatal neurones. Brain Res 217:245-263.

Sternberger LA (1979) Immunocytochemistry. New York: Wiley.

Swanson LW, Cowan WM (1975) A note on the connections and development of the nucleus accumbens. Brain Res 92:324-330.

Terreberry RR, Neafsey EJ (1984) The effects of medial prefrontal cortex stimulation on heart rate in the awake rat. Soc Neurosci Abstr $10: 614$.

Troiano R, Siegel A (1978) Efferent connections of the basal forebrain in the cat: the nucleus accumbens. Exp Neurol 61:185-197.

Voorn P, Jorritsma-Byham B, Van Dijk C, Buijs RM (1986) The dopaminergic innervation of the ventral striatum in the rat: a light- 
and electron-microscopical study with antibodies against dopamine J Comp Neurol 251:84-99.

Voorn P, Gerfen CR, Groenewegen HJ (1989) The compartmental organization of the ventral striatum of the rat: immunohistochemical distribution of enkephalin, substance $P$, dopamine and calcium-binding protein. J Comp Neurol 289:189-201.

Williams MN, Faull RLM (1985) The striatonigral projection and nigrotectal neurons in the rat. A correlated light and electron microscopic study demonstrating a monosynaptic striatal input to identified nigrotectal neurons using a combined degeneration and horseradish peroxidase procedure. Neuroscience 14:991-1010.

Zahm DS, Heimer L (1990) Two transpallidal pathways originating in nucleus accumbens. J Comp Neurol 302:437-446. 